$\begin{array}{cl}\begin{array}{c}\text { Revue } \\ \text { de Ihistoire }\end{array} & \text { Revue de l'histoire des religions } \\ \text { des religions } & \begin{array}{l}\text { 3| } \mathbf{2 0 1 4} \\ \text { Traduction et transmission de la Doctrine dans } \\ \text { l'histoire du bouddhisme }\end{array}\end{array}$

La première traduction coréenne du Sūtra du Lotus (1463)

The first Korean Translation of the Lotus Sūtra (1463)

Kyong-Kon Kim

\title{
OpenEdition
}

Journals

Electronic version

URL: http://journals.openedition.org/rhr/8261

DOI: $10.4000 /$ rhr.8261

ISSN: 2105-2573

Publisher

Armand Colin

Printed version

Date of publication: 1 October 2014

Number of pages: $425-465$

ISBN: 978-2-200-92912-1

ISSN: 0035-1423

Electronic reference

Kyong-Kon Kim, « La première traduction coréenne du Sūtra du Lotus (1463) », Revue de l'histoire des religions [Online], 3 | 2014, Online since 03 November 2014, connection on 20 April 2019. URL : http:// journals.openedition.org/rhr/8261 ; DOI : 10.4000/rhr.8261 


\section{La première traduction coréenne du Sūtra du Lotus (1463)}

Le document coréen Myobŏb ryŏnhhwa'gyŏng, qui contient la première traduction coréenne intégrale du célèbre Miaofalianhuajing de Kumārajīva (344-413) et celle du Miaofalianhuajing yaojie, commentaire du moine Chan chinois Jiehuan (?-1129?), a vu le jour sous le règne du roi Sejo (règ. 1456-1468) de la dynastie Chosŏn, dans l'intention de revitaliser le bouddhisme et d'affaiblir ainsi ses opposants politiques néoconfucéens. Ce document inconnu à l'extérieur de la Corée reflète, cependant, des aspects fondamentaux $d u$ bouddhisme coréen $d u$ $X V^{e}$ siècle, qui perdurent jusqu'à nos jours, notamment la réception quasi systématique de la terminologie bouddhique chinoise, la prédominance de l'esprit æecuménique et la primauté de la voie du Sŏn/Zen.

\section{The first Korean Translation of the Lotus Sütra (1463)}

The Korean document Myobŏb ryŏnhhwa'gyŏng contains the first complete Korean translation of two Chinese texts, the famous Miaofalianhuajing by Kumārajīva (344-413) and the Miaofalianhuajing yaojie, an associated commentary by Jiehuan, a Chan monk from the Song period. This publication was initiated by King Sejo of the Chosŏn dynasty (1392-1910), who intended to revitalize Buddhism in order to weaken his neo-Confucian political opponents. This document, largely unknown outside Korea, reflects some essential aspects of Korean Buddhism in the $15^{\text {th }}$ century which continue to this day, notably the almost systematic reception of the Chinese Buddhist terminology, the predominance of the ecumenical spirit, and the primacy of the Zen way. 
Rares sont des textes religieux qui portent le nom d'une fleur, et le texte bouddhique connu sous le titre abrégé "Le Sūtra du Lotus » en fait partie. Or, ce dernier a souvent été considéré comme l'un des sūtra les plus répandus et les plus lus. Plus précisément, le Miaofalianhuajing 妙法蓮華經 (Sūtra du lotus de la loi merveilleuse, T 262, vol. 9), traduction chinoise réalisée sous la direction du moine centrasiatique Kumārajīva (344-413) en 406 à partir d'une version sanskrite du Saddharmapuṇ̂arīkasūtra (Sūtra du lotus blanc de la vraie doctrine), a rencontré un succès incontestable auprès des lecteurs bouddhistes asiatiques, notamment grâce à sa qualité littéraire, à travers laquelle l'enseignement du Sūtra du Lotus fut exposé dans une beauté stylistique à la fois remarquable et accessible ${ }^{1}$.

Bien qu'il n'y ait guère d'informations sur le texte original indien du Saddharmapuṇ̂rīkasūtra en raison de l'absence des matériaux textuels, nous savons qu'en Chine, le Miaofalianhuajing fut le texte fondateur de l'école Tiantai 天台 (Terrasse céleste), la première des écoles bouddhiques spécifiquement chinoises fondée à la fin $\mathrm{du} \mathrm{VI}^{\mathrm{e}}$ siècle, et qu'il demeura durant les siècles suivants l'un des textes bouddhiques les plus appréciés et les plus révérés. Au Japon, le Miaofalianhuajing (jap. Myōhō renge-kyō), transmis dès la fin $\mathrm{du} \mathrm{VI}^{\mathrm{e}}$ siècle, occupa la place centrale non seulement dans l'école Tendai (chin. Tiantai) instaurée par Saichō (767-822), une des deux écoles principales de l'époque de Heian (794-1185), mais aussi dans l'école Nichiren - en référence au nom de son fondateur Nichiren (1222-1282) issu de l'école Tendai -, qui fut une des quatre écoles principales durant la période de Kamakura (1185/1192-1333). Le Myōhō renge-kyō demeure aujourd'hui encore l'un des sūtra les plus estimés par les bouddhistes japonais, au point d'être considéré comme «une composante essentielle de la culture japonaise $»^{2}$.

\footnotetext{
* Abréviations et symbole $:$ chin. $=$ chinois $;$ cor. $=$ coréen $;$ hun. $=$ hunmin jŏng'ūm; jap. $=$ japonais $;$ pāl. $=$ pāli $;$ skt. $=$ sanskrit $; S Z=$ Shinsan Zokuzōkyō $;$ $\mathrm{T}=$ Taishō shinshū daizōkyō $; \mathrm{Z}=$ Zokuzōkyō $;>=$ devenant.

1. $C f$. Kohler 1962, p. 128 ; Renou et Filliozat 1953, p. 368 ; Frank 2000, p. 235.

2. Robert 1997a, p. 15.
} 
Dans le bouddhisme coréen contemporain, qui se caractérise généralement par la notion de tongbulgyo 統佛㸚 ${ }^{3}$ (bouddhisme œcuménique) ou de hwaŏmsŏn 華嚴禪 ${ }^{4}$ (pratique de méditation basée sur l'étude du Hwaŏmgyŏng), le Myobŏp yŏnhwa'gyŏng (chin. Miaofalianhuajing) figure parmi les quatre sūtra les plus importants avecle Hwaŏmgyŏng (chin.Huayanjing, skt.Avatamsakasūtra, Sūtra de l'ornementation fleurie), le Gümgang'gyŏng (chin. Jingangjing, skt. Vajracchedikāprajñāpāramitāsūtra, Sūtra du diamant trancheur de la perfection de sagesse) et le Nüng'ga'gyŏng (chin. Lengqiejing, skt. Lañkāvatārasūtra, Sūtra de l'entrée à Lan்ka) ${ }^{5}$. C'était vraisemblablement déjà le cas durant la dynastie Chosŏn (1392-1910), car le Myobŏp yŏnhwa'gyŏng faisait partie des sūtra les plus imprimés dans la péninsule coréenne entre 1236 et 1876 , avec ses cent six différentes impressions ${ }^{6}$. En outre, après la création du hunmin jŏng'ūm (phonie correcte pour l'instruction du peuple) en 1446, le Miaofalianhuajing de Kumārajīva fut sélectionné trois fois - en 1447, en 1459 et en 1463 -, afin d'être traduit en cette écriture coréenne prémoderne. Parmi ces trois versions, celle de l'année 1463 mérite une attention particulière, car ce Myobŏb ryŏnhhwa'gyŏng 妙法蓮華經 (=MBRHG), appelé conventionnellement Bŏphwa'gyŏng ŏnhae (Traduction du Sūtra de fleur de la loi), demeure la première traduction intégrale du Miaofalianjuajing en langue coréenne. Par ailleurs, le MBRHG comprend également une traduction coréenne complète du Miaofalianhuajing yaojie 妙法蓮華經要解 ${ }^{7}$ (Compendium $d u$ Sütra du lotus de la loi merveilleuse, SZ 602, vol. 30) de Jiehuan 戒環 (?-1129?), moine chinois de l'école Chan. Ce dernier document, quasi inconnu sur le continent chinois et dans l' archipel japonais, est devenu le commentaire par excellence du Miaofalianhuajing dans la péninsule coréenne durant la dynastie Chosŏn : parmi les cent six versions imprimées du Miaofalianhuajing, nous pouvons constater qu'au moins cinquante versions contiennent le commentaire de Jiehuan.

3. $C f$. PARK 1999 , p. 60 ; RHI 2003, p. 51.

4. $C f$. KYONG'IL 1987, p. 54.

5. Cf. KIM, Y.-T., 1977, p. 5.

6. Cf. Ko 1987, p. 83-89: il a été imprimé deux fois durant la dynastie Koryŏ, et cent quatre fois durant la dynastie Chosŏn.

7. Cf. Huang 2011. 
À l'heure actuelle, aucune étude n'a été menée sur le MBRHG en dehors de la Corée, tandis qu'au pays du Matin calme, les recherches ont porté essentiellement sur trois domaines. D'abord, l'étude bibliographique a permis de rétablir une chronologie des trois traductions coréennes du Miaofalianhuajing de Kumārajīva, ainsi que celle de diverses éditions postérieures réalisées sur la base de la xylographie du $\mathrm{MBRHG}^{8}$. Puis, grâce à la recherche linguistique, il a été démontré que seul le MBRHG contient une traduction intégrale du Miaofalianhuajing, les deux traductions précédentes restant partielles ou sélectives, et que le style littéraire du MBRHG est plutôt littéral, c'est-à-dire soucieux d'offrir une traduction plus précise et exacte que la traduction de 1447 pouvant être considérée comme plus indigène et libérale ${ }^{9}$. Enfin, observant la quantité imposante de la première traduction du Miaofalianhuajing insérée dans l'ouvrage Syŏgbo ssangjyŏl (Biographie de Śākyamuni en prose, 21 vol., 1447) - neuf volume sur vingt et un -, et celle de la deuxième insérée dans l'ouvrage Wǒlin syŏgbo (composé du Wǒlin chŏnkang jigok [Chant de la lune brillant sur les mille rivières, 1449] et du Syŏgbo ssangjyŏl, 19 vol., 1459) - neuf volumes sur dix-neuf -, ainsi que l'importance du MBRHG, le deuxième ouvrage publié après le Nūngga'gyŏng (skt. Lañkāvatārasūtra) par le Gan'gyŏng do'gam (Institut pour la publication des sūtra), les bouddhologues en ont déduit que les trois traductions coréennes du Miaofalianhuajing furent probablement réalisées sur la base de la notion du gyopan (chin. jiaopan, classification critique des doctrines) de l'école Chŏntae (chin. Tiantai) considérant le Sütra $d u$ Lotus comme le texte suprême sur le plan doctrinal. Ces mêmes chercheurs ont également supposé que le Miaofalianhuajing yaojie, le commentaire de Jiehuan, moine chinois de l'école Chan de la période des Song (960-1279), aurait été intégré dans le MBRHG en raison de son point de vue doctrinal qui s'accordait avec le courant principal de pensée des bouddhistes coréens du début de la dynastie Chosŏn ${ }^{10}$.

Néanmoins, aucun bouddhologue ne s'est intéressé à la question de la réception-évolution linguistique des termes techniques boud-

8. Cf. AN $1971 ; 1998$.

9. $C f$. Kim et NaKamura 2010, p. 19-28.

10. Cf. Ko 1987, p. 102-110; KIM, Y.-G., 1997; KANG 1997. 
dhiques dans le MBRHG, et peu sont ceux qui ont consacré leur recherche à l'identification des éléments communs entre le point de vue de Jiehuan, exposé dans le Miaofalianhuajing yaojie, et la vision bouddhique des Coréens du $\mathrm{Xv}^{\mathrm{e}}$ siècle $^{11}$. Il s'avère donc intéressant d'interroger ces diverses sources afin de mieux saisir comment les termes techniques bouddhiques indiens, traduits en chinois dans le Miaofalianhuajing, ont été réceptionnés dans cette langue coréenne prémoderne et d'identifier ainsi les particularités doctrinales du Miaofalianhuajing yaojie de Jiehuan qui ont permis à ce dernier d'être intégré dans le MBRHG. Dans cet article, nous nous consacrerons principalement au volume I du MBRHG contenant les chapitres I et II, qui est considéré comme le noyau du Sūtra du Lotus, et dans lequel se trouvent des explications précieuses de Jiehuan. Ainsi, il sera possible par l'étude de ce document inédit qui exprime quelques aspects essentiels de la pensée bouddhique coréenne, de (ré)évaluer sa portée religieuse dans l'histoire plus générale du bouddhisme coréen.

\section{LE Sütra dU LOTUS DANS LES CONTEXTES INDIEN, CHINOIS ET CORÉEN}

\section{Le Saddharmapuṇ̣arīkasūtra dans le saṃgha indien ${ }^{12}$}

Le MBRHG de l'année 1463 est une traduction du Miaofalianhuajing, qui est lui-même un texte traduit à partir d'une version sanskrite du Saddharmapuṇ̂räkasūtra rédigé au sein du saṃgha (pāl. sañgha, communauté bouddhique) indien dont l'origine remonte au Buddha (l'Éveillé) Śākyamuni (l'ascète [ayant fait vœu de silence du clan] des Śākya), personnage historique mais dont la figure de fondateur a été construite au cours des siècles par ses fidèles selon les enjeux doctrinaux et les nécessités qui se sont imposés à eux. L'essentiel de l'enseignement du Buddha Śākyamuni, qui promulgua son dharma (pāl. dhamma, doctrine)

11. Cf. Ko 1987, p. 83-121.

12. Cf. Vallée Poussin 1909; Przyluski 1932; Conze 2002 (1952), p. 119227; Renou et Filliozat 1953, p. 463-586; Bareau 1955a; Bareau 1955b; Lamotte 1958; Bareau 1966; Bareau 1970; Hirakawa 1990; Harvey 1993 ; ROBERT 1993, p. 499-529; ROBERT in Encyclopaedia Universalis; BRONKHORST 2007; DuCEur 2011. 
approximativement au $\mathrm{V}^{\mathrm{e}}$ siècle av. J.-C. et principalement dans le bassin moyen du Gange, aurait reposé sur les quatre nobles vérités (skt. caturāryasatya, pāl. cattāro ariyasaccāni) et la loi de la production conditionnée (skt. pratītyasamutpāda, pāl. pațiccasamuppāda) : une voie de salut différente de celle du sacrifice rituel des brāhmaṇa et de celles enseignées par d'autres courants śramaṇiques tels que les jaïna, les àjīivika, les matérialistes, les sceptiques, etc.

Le samgha indien, qui connut déjà au temps du Buddha des divergences doctrinales et disciplinaires, fut marqué par un schisme au concile (skt. samgīti, pāl. sangīiti, récitation en chœur) de Pātaliputra dont la date demeure incertaine. En raison du désaccord sur l'arhattva (état d'arhant) ou sur les nouvelles règles disciplinaires, le saṃha se divisa en deux grandes parties: les réformateurs de l'arhattva majoritaires, nommés de ce fait Mahāsāṃghika (ceux de la grande assemblée), et les moines opposants minoritaires de tendance rigoriste et fidèles à l'antique rigueur, les Sthaviravādin (ceux qui professent [la doctrine] des Anciens). Puis, durant les siècles suivants, des divisions internes se produisirent au sein de ces deux courants et donnèrent progressivement naissance à une vingtaine d'écoles (skt. nikāya, pāl. ācariyavāda) qui élaborèrent chacune leur propre canon, le tripițaka (pāl. tipițaka, trois corbeilles).

Vers le II ${ }^{\mathrm{e}}$ siècle av. J.-C., au sein des écoles des Mahāsāṃghika, émergea lentement une nouvelle forme de pratique bouddhique influencée par le florissant courant de la bhakti (dévotion envers une divinité). Nommée d'abord bodhisattvayāna (véhicule de l'être en marche vers l'Éveil), cette nouvelle doctrine bouddhique prendra ensuite l'appellation de mahāyāna (grand véhicule). Cette évolution fut accompagnée par un mouvement laïc, qui mit en avant la vénération des stūpa (reliquaires) du Buddha, et qui s'opposa aux excès du monachisme aristocratique et de la scolastique abhidharmique. Ainsi, ces laïcs prônaient-ils une autre voie de salut que celle de l'arhattva suivie par les bhikṣu et les bhikșunī. Cette réforme bouddhique, qui redéfinit la nature même du Buddha et des bodhisattva, en faisant une large place à la notion de súnyatā (vacuité), eut pour conséquence d'ouvrir la pratique religieuse à un plus grand nombre de personnes, d'accorder un rôle plus important aux laïcs et d'étendre à tous les êtres la possibilité d'atteindre la délivrance.

Ce mouvement mahāyānique s'accompagna également de la rédaction de nouveaux sūtra, appelés souvent vaipulyasūtra (sūtra 
développés) en raison de leur forme étendue et rédigés en sanskrit langue sacrée de l'Inde védique et brāhmaṇique dont le bouddhisme ancien avait refusé l'usage -, dans l'intention d'affirmer sa nouvelle doctrine et de supplanter ainsi l'enseignement des tripitaka. Les rédacteurs mahāyānistes présentèrent leurs sūtra comme des paroles authentiques du Buddha, qui professaient un enseignementésotérique réservé seulement aux êtres susceptibles de le comprendre, et qui révélaient l'accessibilité pour tous à la bouddhéité. Néanmoins, ce nouveau courant bouddhique ne rejeta ni le śrāvakayāna (véhicule de l'auditeur) ni le pratyekabuddhayāna (véhicule des buddha solitaires). Il se présenta plutôt comme l'accomplissement de ces deux véhicules et s'autoproclama, de ce fait, ekayāna, c'est-à-dire véhicule unique. Le corpus essentiel des textes mahāyāniques, tels que le Prajñāpāramitāsūtra (Sūtra de la perfection de sagesse), le Saddharmapuṇ̣̂īikasūtra, l'Avatamsakasūtra (Sütra de l'ornementation fleurie), le Sukhāvatīvyūhasūtra (Sūtra du développement sur l'heureuse [terre]), le Suvarnaprabhāsa (Éclat d'or), le Vimalakìrtinirdeśasūtra (Sütra de l'enseignement de Vimalakìrti), etc., fut achevé vers le $\mathrm{II}^{\mathrm{e}}$ siècle ap. J.-C. Le texte original du Saddharmapuṇ̣̂inkasūtra, qui serait conservé dans les vingt premiers chapitres sur les vingt-sept du texte fixé, aurait été composé en plusieurs étapes, entre le $\mathrm{I}^{\mathrm{er}}$ siècle av. J.-C. et le milieu du $\mathrm{I}^{\text {er }}$ siècle ap. J.-C., initialement en prakṛt puis réécrit ultérieurement en un sanskrit hybride par un travail de transposition de ces bouddhistes mahāyānistes ${ }^{13}$.

Le Saddharmapundarīkasūtra qui porte sur le saddharma (vraie doctrine) - il s'agit vraisemblablement de la doctrine de l'ekayāna, de l'accessibilité universelle à la bouddhéité et de l'existence éternelle du Tathāgata (Ainsi-venu/allé) - fut l'objet de réflexions de la part des générations suivantes de bouddhistes indiens, notamment ceux ayant appartenu aux deux grandes écoles mahāyāniques. Nāgārjuna (II - III ${ }^{\mathrm{e}}$ s. ap. J.-C.), fondateur de l'école Madhyamaka (voie moyenne), cita ce sūtra une trentaine de fois dans son ouvrage Traité de la grande vertu de sagesse, commentaire monumental du Mahāprajñāpāramitāsūtra, qui n'existe plus que dans sa version chinoise intitulée le Dazhidulun (T 1509, vol. 25,

13. $C f$. Fujita 1980; Tamura 1989; Jong 1986; Hirakawa 1996; Мототsuki 1996; Fuss 1991; Suh 1997; Tsuкamoto 2010. 
skt. Mahāprajñāpāramitopadeśa). Il aurait également rédigé un Saddharmapuṇdarīkaśāstra (Traité du Sūtra du lotus de la vraie doctrine; chin. Fahualun, Traité [du Sūtra] de fleur de la loi), mais ce dernier n'est pas parvenu jusqu'à nos jours. Vasubandhu (320-400?) de l'école des Vijñānavādin (ceux qui professent [la doctrine de] la conscience) ou Yogācārin (ceux qui pratiquent le yoga) composa un commentaire du Saddharmapuṇaraikasūtra en deux volumes, conservé grâce à deux versions chinoises intitulées le Miaofalianhuajing youpotishe (Traité du Sütra du lotus de la loi merveilleuse, T 1519 et 1520 , vol. 26), dont le titre original sanskrit aurait dû être le Saddharmapuṇarīkopadeśa. C'est le seul commentaire indien du Saddharmapundarīkasūtra transmis jusqu'à nos jours, dont l'influence sur les commentaires chinois du même sūtra de générations suivantes fut considérable ${ }^{14}$. Quant aux versions sanskrites du Saddharmapuṇ̣arịkasūtra des premiers siècles de l'ère chrétienne, elles ont disparu en Inde. Néanmoins, depuis le XIX ${ }^{\mathrm{e}}$ siècle, pas moins de trente-six versions sanskrites du Saddharmapuṇ̂rīkasūtra ont été retrouvées au Népal, à Gilgit au Kaśmīr et à Bāmiyān en Afghanistan, dont les plus anciennes datent au plus tôt du $\mathrm{VI}^{\mathrm{e}}$ siècle ap. J.-C. Par conséquent, nous ne pouvons plus guère savoir à quoi ressemblait le Urtext du Saddharmapuṇ̣̂īkasūtra, à l'exception de son titre sanskrit et de son existence avérée avant 286 ap. J.-C., date à laquelle Dharmarakșa réalisa sa traduction chinoise intitulée le Zhengfahuajing 正法華經 (Sūtra de fleur de la loi juste, T 263, vol. 9).

\section{Le Fahuajing dans le bouddhisme chinois ${ }^{15}$}

Le bouddhisme, qui se propagea en dehors du subcontinent indien dès l'époque d'Aśoka (règ. 268-232 av. J.-C.), fut introduit en Chine probablement, au plus tard, au $\mathrm{I}^{\mathrm{er}}$ siècle ap. J.-C. par des moines qui empruntèrent des voies commerciales. Dès lors, cette religion étrangère nouvellement implantée fut perçue comme un

14. $C f$. Nomura 1996; Maruyama 1996.

15. $C f$. MasPero 1950 , p. $65-83$, 195-211; Fong 1952, p. 251-263; RenOU et Filliozat 1953, p. 398-463; Wright 1959; ZürCHer 1959, p. 18-80; Demiéville 1970, p. 1249-1319; Demiéville 1973a, p. 22 et 1258; Demiéville 1973b, p. 212-216; Kim, I.-T., 1987, p. 32-43; Magnin 2003, p. 411-469; LeE, Y.-J., 2003, p. 96-152. 
dérivé du taoïsme en raison des similitudes apparentes des pratiques méditatives et de certains concepts. Les premiers bouddhistes chinois convertis réclamèrent alors la traduction d'ouvrages bouddhiques présentant des exercices mentaux et respiratoires. Ainsi, AN Shigao, prince parthe arrivé à Luoyang en 148, ouvrit-il l'ère de la traduction des textes bouddhiques en chinois. Cependant, durant cette période d'introduction du bouddhisme en Chine ( $\mathrm{I}^{\mathrm{er}}-\mathrm{III}^{\mathrm{e}}$ s. ap. J.-C.), les traducteurs durent emprunter au taoïsme son vocabulaire en raison d'un manque de références linguistiques dû en grande partie aux différences culturelles et épistémologiques. Le Zhengfahuajing, la plus ancienne traduction connue du Sūtra du Lotus, réalisée en 286 sous la direction de Dharmarakșa, en est un exemple.

Avec la conversion de l'élite chinoise au cours des siècles suivants ( $\mathrm{IV}^{\mathrm{e}}-\mathrm{VI}^{\mathrm{e}}$ s. ap. J.-C.), de nouvelles traductions des textes bouddhiques indiens, libérées de toute teinte taoïste, furent exigées. Au début du $v^{\mathrm{e}}$ siècle, le travail de traduction marqua un progrès important grâce à la collaboration entre des érudits indiens ou centrasiatiques connaissant le sanskrit et le chinois et des lettrés chinois. Ainsi virent le jour les grandes traductions réalisées sous la direction de Kumārajīva (344-413), dont celle du Saddharmapuṇ̣̂īkasūtra intitulée le Miaofalianhuajing (Sütra $d u$ lotus de la loi merveilleuse, T 262, vol. 9) en 406. Désormais, ce dernier demeurera une œuvre incontournable dans l'histoire du bouddhisme chinois, voire extrême-oriental. En outre, une autre traduction sous le titre du Tianpin miaofalianhuajing 添品妙法 蓮華經 (Complément du Sūtra du lotus de la loi merveilleuse, T 264, vol. 9), réalisée deux cents ans plus tard, en 601, par Jñānagupta et Dharmagupta, s'appuya entièrement sur la version de Kumārajīva. Les trois traductions chinoises du Sūtra du Lotus, conservées jusqu'à nos jours, furent ainsi terminées avant le début $\mathrm{du} \mathrm{VII}^{\mathrm{e}}$ siècle. Par ailleurs, les recherches récentes sur les manuscrits sanskrits retrouvés ont permis d'estimer que si Dharmarakșa avait probablement effectué sa traduction à partir d'une des versions centrasiatiques, Kumārajīva, quant à lui, aurait réalisé la sienne en prenant comme texte de départ l'une des versions népalaises ${ }^{16}$.

Grâce à ces nouvelles traductions des sūtra et des ouvrages abhidharmiques issus des deux grandes écoles mahāyāniques, le 
Madhyamaka et le Vijñānavāda-Yogācāra, le bouddhisme put prendre son essor en Chine et devenir une religion autonome. Les bouddhistes chinois commencèrent alors à s'approprier les doctrines bouddhiques indiennes et à élaborer des traités originaux. Les deux disciples de Kumārajīva, Daosheng (355-434) et Huiguan (424453), rédigèrent ainsi chacun un commentaire du Sūtra du Lotus, intitulé respectivement le Miaofalianhuajingshu (Traité du Sūtra du lotus de la loi merveilleuse, SZ 577, vol. 27) et le Fahuajing zongyaoxu (Préface du Compendium du Sūtra de fleur de la loi, T 2145, vol. 55). Le commentaire de Fayun (467-529), le Fahuajing yiji (Sens du Sütra de fleur de la loi, T 1715, vol. 33), qui résuma les messages fondamentaux du Sūtra du Lotus par l'intermédiaire des notions de «kaisan xianyi kaijin xianyuan» (ouvrant les trois, faire apparaître le un; ouvrant le proche, faire apparaître le loin), et qui exerça une influence considérable sur les ouvrages du fondateur réclamé de l'école Tiantai, Zhiyi (531-597), date également de cette période.

Durant les dynasties des Sui (581-618) et des Tang (618-907), l'apogée du bouddhisme en Chine, une sinisation ou réinterprétation du bouddhisme indien sur la base de la pensée chinoise, notamment confucéenne et taoïque, fut entreprise. Quant au travail de traduction, son niveau s'éleva à un haut degré de perfection. Dès lors, différentes écoles bouddhiques chinoises furent fondées et affirmèrent leur légitimité par leur interprétation de la doctrine et par la lignée de leurs patriarches. L'école Tiantai ${ }^{17}$ - toponyme du mont où Zhiyi enseigna -, qui vit le jour à la fin du $\mathrm{VI}^{\mathrm{e}}$ siècle, fut la première des écoles bouddhiques spécifiquement chinoises à revendiquer comme texte fondateur le Sütra du Lotus. Zhiyi, qui systématisa les doctrines de l'école, composa un grand nombre d'ouvrages, dont trois furent considérés comme les plus importants: le Miaofalianhuajing wenju (Explication textuelle du Sūtra du lotus de la loi merveilleuse, 20 vol., 587; T 1718, vol. 34), le Miaofalianhuajing xuanyi (Sens profond du Sūtra du lotus de la loi merveilleuse, 20 vol., 593; T 1716, vol. 33) et le Mohe zhiguan (Grandes concentration et contemplation, 20 vol., 594; T 1911, vol. 46). Il élabora un système herméneutique appliquant la notion de fangbian (skt. upāyakauśalya, expédients salvifiques), exposée

17. Cf. Petzold 1987, p. 3-12. 
dans le Sütra du Lotus, au jiaopan (classification critique des doctrines): en considérant l'enseignement de tous les sūtra comme vérité adaptée aux capacités des auditeurs selon les différentes périodes de prédication du Buddha - le wushibajiao (huit doctrines [révélées] en cinq périodes) -, l'enseignement du Sūtra du Lotus fut placé en tant qu'aboutissement de tout enseignement du Buddha. L'école Tiantai devint pendant cette période une des écoles les plus populaires en Chine, et son texte fondateur, le Sütra du Lotus, exerça une influence considérable sur toutes les écoles bouddhiques chinoises. Par exemple, Jizang (549-623) de l'école Sanlun (Trois traités) fondée sur l'abhidharma de Nāgārjuna et d'Āryadeva, rédigea à son tour des commentaires sur le Sūtra du Lotus : le Fahua xuanlun (Discours profond sur [le Sūtra de] fleur de la loi, T 1720, vol. 34), le Fahua yishu (Commentaire sur [le Sütra de] fleur de la loi, T 1721, vol. 34) et le Fahua youyi (Quelques pensées sur [le Sütra de] fleur de la loi, T 1722, vol. 34). Guiji (632-682), disciple de Xuanzang (602-664), grand traducteur et fondateur de l'école Faxiang (Caractéristiques de la loi) apparentée au VijñānavādaYogācāra indien, écrivit pour sa part le Miaofalianhuajing xuanzan (Éloge du Sütra du lotus de la loi merveilleuse, T 1723, vol. 34). Ceci montre combien le Sūtra du Lotus joua un rôle considérable dans le bouddhisme chinois au-delà même de l'école Tiantai ${ }^{18}$.

Après des siècles de prospérité, durant lesquels les confucéens n'hésitèrent pas à manifester leur hostilité envers un saṃgha qu'ils dénoncèrent comme étant trop puissant économiquement et marqué par l'opulence et le goût du luxe, la dernière et la plus importante proscription de 845 amorça le début d'une longue période de déclin du bouddhisme en Chine. En même temps, cette circonstance ouvrit le saṃgha chinois à un temps d'évolution marquée par un syncrétisme ou œcuménisme ad intra et ad extra. À l'intérieur du saṃgha, s'opéra une sorte de fusion entre les quatre principales écoles bouddhiques: sur le plan exégétique, l'école Tiantai et l'école Huayan, et sur le plan pratique, les méthodes méditatives de l'école Chan et la pratique commémorative envers le Buddha Emituo (skt. Amitābha/Amitāyus, Lumière/Vie infinie) - nianfo - et de multiples bodhisattva de l'école Jingtu (Terre pure). Progressivement, les écoles scolastiques s'effacèrent, tandis que les pratiques de Chan

18. Cf. Shioniri 1996, p. 227; Lee, Y.-J., 1998, p. 28-31. 
et celles de Jingtu se maintinrent et se rapprochèrent au cours des siècles suivants. Vers l'extérieur du saṃgha, une tendance syncrétique se manifesta afin de contrecarrer les attaques des néoconfucéens. Des moines lettrés s'efforcèrent de trouver des éléments communs entre le bouddhisme et le confucianisme et de concilier les trois doctrines, notamment le confucianisme, le bouddhisme et le taoïsme. Jiehuan (?-1129?) de l'école Chan, l'auteur du Miaofalianhuajing yaojie (Compendium du Sūtra du lotus de la loi merveilleuse, 7 vol., 1126; SZ 602, vol. 30) intégré dans le MBRHG coréen de l'année 1463, vécut justement à la période des Song (960-1279), durant laquelle les tendances syncrétiques et œcuméniques du saṃgha se généralisa. Séjournant au temple Wenling kaiyuanliansi de la branche Huanglong de l'école Linji, Jiehuan rédigea également deux autres ouvrages: le Lengyanjing yaojie (Compendium du Śūraṃgamasūtra, 1128; SZ 270, vol. 11) et le Huayanjing yaojie (Compendium d'Avatamsakasūtra, 1129; SZ 238, vol. 8).

\section{Le Bø̆phwa'gyŏng dans la péninsule coréenne ${ }^{19}$}

De Chine, le bouddhisme se répandit dans la péninsule coréenne durant la période des Trois Royaumes coréens et fut assez rapidement reconnu officiellement dans chacun des trois royaumes. Il devint religion d'État, à la fin du IV siècle, aux royaumes de Koguryŏ (37 av. J.-C.-668 ap. J.-C.) et de Baegje (18 av. J.-C.-660 ap. J.-C.), et fut officiellement reconnu au royaume de Silla (57 av. J.-C.-668 ap. J.-C.) en 534. Deux raisons ont été avancées quant à ces reconnaissances et à cette implantation. Il est probable que les dirigeants des Trois Royaumes aient adopté le bouddhisme pour des raisons d'intérêts politiques externes et internes afin, d'une part, d'entretenir de bonnes relations diplomatiques avec son puissant voisin chinois, et d'autre part, de maintenir une cohésion sociale, c'est-à-dire unifier et centraliser le peuple sur un plan idéologique. Les peuples des Trois Royaumes semblent avoir aisément adopté cette nouvelle religion en raison des points communs avec le

19. Cf. Kim, H.-J., 1958; Li 1969; Vos 1977; Ho 1978; Kıм, U.-T., 1981; Keel 1984; Kamata 1994; Lee, Y.-J., 1998; Park 1999; Buswell 1999; Sørensen 1999; Fabre 2000 ; Jogye Order 2004; Lee, E.-J., 2005 ; Kim, K.-K., 2007, p. $14-88$. 
chamanisme, croyance prédominante dans la péninsule d'alors. En ce qui concerne le Sütra du Lotus, seuls quelques documents étrangers permettent de supposer son introduction dans la péninsule durant cette période. Selon le document japonais Shōtoku taishi denryaku (Relation du prince Shōtoku), le prince japonais Shōtoku (574-622), l'auteur du Hokke gi-sho (Commentaire [du Sūtra] de fleur de la loi, T 2187, vol. 56), aurait discuté sur le Sūtra du Lotus avec son maître Haeja venu du royaume de Koguryŏ. Deux documents chinois, le Song gaosengchuan (Relation des moines de la dynastie des Song, 998; T 2061, vol. 50) et le Fozutongji (Chronique des patriarches bouddhistes, 1269; T 2035, vol. 49), indiquent qu'un moine de Baegje nommé Hyŏn'gwang, après avoir étudié le Sūtra du Lotus auprès de Huisi (515-577), le deuxième patriarche de l'école Tiantai chinoise, serait retourné dans son pays, et que Yŏn'gwang, moine venu du royaume de Silla, serait devenu disciple de Zhiyi, le troisième patriarche et fondateur réclamé de l'école Tiantai. De ce fait, il est fort possible que le Sütra du Lotus ait été introduit et étudié dans la péninsule coréenne durant la période des Trois Royaumes.

En 668 , le royaume Silla parvint à conquérir les deux autres royaumes, et une nouvelle période nommée Silla Unifié (668918) commença. Ce fut aussi l'âge d'or du bouddhisme coréen, durant lequel ce dernier devait jouer un rôle tutélaire du pays vis-à-vis des invasions étrangères par le biais de divers rituels. Parallèlement, des doctrines bouddhiques se systématisèrent dans cinq écoles scolastiques: l'école Gyeyul (Disciplines), l'école Yŏlban (Extinction), l'école Bŏpsang (Caractéristique de la loi), l'école Hwaŏm (Ornementation fleurie) et l'école Bŏpsŏng (Nature de la loi). L'école Hwaŏm, fondée par Ūisang (625-702) et basée sur le Hwaŏmgyŏng (chin. Huayanjing) soulignant la notion de l'interdépendance et de l'interpénétration de tous les êtres ainsi que le concept de yŏraejang (chin. rulaicang, skt. tathāgatagarbha, embryon de l'Ainsi-venu/allé), fut appréciée par la noblesse et devint l'école la plus répandue dans le royaume. Dès cette époque, l'étude du Hwaŏmgyŏng prit une place centrale dans la scolastique bouddhique. L'esprit de synthèse et d'unification harmonieuse de différentes écoles bouddhiques, la pensée de hwajaeng 和猙 (harmonisation des éléments conflictuels) de Wŏnhyo (617686), le fondateur de l'école Bŏpsŏng, la seule école bouddhique 
spécifiquement coréenne parmi les cinq, restera dès lors l'idée axiale du bouddhisme coréen. Egalement, l'école Chan (cor. Sŏn) put-elle s'introduire dès la deuxième moitié du $\mathrm{VII}^{\mathrm{e}}$ siècle et se propagea, entre le $\mathrm{IX}^{\mathrm{e}}$ et le $\mathrm{X}^{\mathrm{e}}$ siècle ap. J.-C., dans toute la péninsule. Du fait que la majorité des maîtres Sŏn coréens étaient des disciples du maître chinois Mazu Daoyi (709-788), dans la lignée du sixième patriarche de l'école Chan chinoise, Huineng (638-713), les écoles Sŏn, connues sous la dénomination collective de Gusan sŏnmun (portes de méditation de neuf montagnes), s'inscrivirent dans le courant de pensée de la primauté de l'expérience d'Éveil directe et subite sur l'étude scolastique. En outre, les différentes approches vers l'Éveil, proposées par les deux grands courants bouddhiques - le gyojong (école scolastique) et le sŏnjong (école méditative), appelés collectivement Ogyogusan (cinq doctrines et neuf montagnes) -, furent d'une certaine manière à l'origine des conflits et des divisions entre les écoles bouddhiques que subira le saṃgha coréen à l'avenir, surtout durant la dynastie Koryŏ (9181392). Par ailleurs, il est à noter qu'à cette époque, la doctrine de l'école Tiantai chinoise ne donna naissance à aucune école bouddhique coréenne, même s'il est attesté que sept auteurs rédigèrent environ treize ouvrages sur le Sūtra du Lotus, dont deux sont conservés: le Bŏphwa jong'yo (Compendium [du Sūtra] de fleur de la loi, T 1725, vol. 34) de Wŏnhyo (617-686) et le volume I du Bŏphwa'gyŏng nonsulgi (Traité du Sūtra de fleur de la loi, Z série 1, vol. 95, fascicule 4) de Üijŏk (?-?). Le fait que Wŏnhyo, le moine le plus important de cette période, rédigea des ouvrages sur le Sütra du Lotus, peut non seulement prouver la présence du Sūtra du Lotus dans le royaume de Silla Unifié mais aussi permettre d'estimer la place que le même sūtra aurait pu occuper au sein du samigha coréen.

Durant la dynastie Koryŏ (918-1392), le bouddhisme demeura religion d'État assurant pleinement son rôle tutélaire du pays en proie à plusieurs invasions étrangères importantes. Dans l'espoir d'obtenir la bénédiction et la protection du Buddha, le pouvoir royal fit construire des temples selon les règles géomantiques. Pour la même raison, l'édition xylographique de l'immense Canon bouddhique fut réalisée à deux reprises: la première entre 1019 et 1087, et la deuxième entre 1236 et 1251 . Sous protectorat royal, le bouddhisme de Koryŏ poursuivit également son indigénisation dans 
l'esprit de synthèse et d'unité qu' avait propagé Wŏnhyo du royaume de Silla Unifié. Par exemple, Ūichŏn (1055-1101), le fondateur de l'école coréenne Chŏntae (chin. Tiantai), témoigna d'un réel souci d'harmonie entre les écoles bouddhiques et tenta de mettre un terme à la rivalité entre le gyojong (école scolastique) et le sŏnjong (école méditative connue sous le nom de Jogye), tout en accordant une préférence à l'étude scolastique. Même si la volonté de Ūichŏn ne put aboutir à cause de son décès prématuré, l'école Chŏntae prospéra jusqu'à la fin de la dynastie Koryŏ. Une centaine d'années plus tard, Chinul (1158-1210) reprit l'initiative d'unifier les différentes écoles bouddhiques dans un esprit d'harmonisation. Principalement, il mit en avant la pratique du ganhwasŏn (chin. jianhuachan, méditation par la contemplation de hwadu [chin. huatou, mot-clef d'un gongan]) comme méthode suprême vers l'Éveil tout en proposant d'autres chemins selon les facultés de chacun: la voie graduelle qui débute par l'étude scolastique du Hwaŏmgyŏng pour les pratiquants munis de facultés moyennes, ou la voie du yŏmbul (chin. nianfo) pour les pratiquants aux facultés moindres. Cette pluralité de pratiques vers l'Éveil, initiée par Chinul, s'imposa progressivement comme modèle dans le bouddhisme coréen. De plus, Chinul essaya de mettre en œuvre son enseignement par le biais du mouvement du Jŏnghye gyŏlsa (Assemblée [pour la pratique] de concentration et sagesse). Dans la même période, Yose (1163-1245), le deuxième patriarche de l'école Chŏntae, fonda le Baegryŏn gyŏlsa (Assemblée du lotus blanc) et visa à renouveler et à approfondir la spiritualité du peuple bouddhiste en s'appuyant sur l'enseignement du Sūtra du Lotus, la foi en la renaissance dans la Terre pure et la pratique de ji'gwan (chin. zhiguan, concentration et contemplation). Dès cette époque, le mouvement du Baegryŏn gyŏlsa de l'école Chŏntae s'ouvrit au peuple, tandis que le mouvement du Jŏnghye gyŏlsa de Chinul fut réservé plutôt au milieu élitiste. Par ailleurs, les recherches sur l'ouvrage de Chegwan (?-970), le Chŏntae sa'gyo'üi (Disposition des quatre doctrines de la Terrasse céleste, T 1931, vol. 46) ${ }^{20}$, nous amènent à penser que la doctrine de l'école Tiantai chinoise fut déjà étudiée dans la péninsule coréenne avant même la fondation de l'école Chŏntae par Ūichŏn en 1097. Néanmoins, ce bouddhisme, protégé par le pouvoir royal, suscita de nombreuses critiques de la

20. $C f$. Kim, D.-J., 1984; Robert 2007, p. 329-397. 
part des lettrés néoconfucéens qui décrièrent les excès de rituels et la vie opulente des moines ainsi que leur implication de plus en plus croissante dans la vie politique.

L'avènement de la nouvelle dynastie Chosŏn (1392-1910) adoptant le néoconfucianisme comme principe de gouvernement marqua donc pour le bouddhisme coréen le début d'une longue période d'oppression et de restriction. Par exemple, le roi Sejong, le plus brillant de toute histoire de Corée qui régna de 1418 à 1450, prit des mesures drastiques contre le samgha: seuls trente-six monastères furent autorisés dans le pays; la construction de nouveaux monastères fut interdite; une réforme du clergé bouddhique fut mise en place permettant le contrôle de la majeure partie des terres du samgha par l'État, etc. Pourtant, ce fut le même roi qui délégua au prince Suyang'gun, le futur roi Sejo, la direction de l'édition de l'ouvrage Syŏgbo ssangjyŏl (Biographie de Śäkyamuni en prose, 21 vol., 1447), l'un des premiers ouvrages publiés en hunmin jŏngūm (phonie correcte pour l'instruction du peuple) $)^{21}$, l'écriture coréenne prémoderne promulguée en 1446. La toute première traduction coréenne du Miaofalianhuajing de Kumārajīva y occupa les volumes XIII à XXI. Il s'agissait alors d'une traduction sélective des parties soit en prose soit en vers de chacun des chapitres du Miaofalianhuajing accompagnée d'un bref commentaire explicatif des termes bouddhiques. Sous le règne du roi Sejo (1456-1468), le bouddhisme vécut un moment de revitalisation. En essayant de protéger et de répandre le bouddhisme, le roi Sejo voulut non seulement instruire davantage le peuple bouddhiste, mais aussi consolider son pouvoir politique grâce au soutien des nombreux bouddhistes et ainsi affaiblir ses opposants, les lettrés néoconfucéens. Le roi Sejo ordonna alors la traduction et la publication d'un grand nombre de textes bouddhiques, afin de présenter la vie du Buddha et de familiariser le peuple avec le tripitaka ${ }^{22}$. Ainsi, fut publié l'ouvrage intitulé le Wŏlin syŏgbo, composé du Wŏlin chŏnkang jigok (Chant de la lune brillant sur les mille rivières, 1449) et du Syŏgbo ssangjyŏl, en 1459 en dix-neuf volumes, parmi lesquels la nouvelle et deuxième traduction coréenne du Miaofalianhuajing fut insérée dans les volumes XI à XIX. Il s'agissait encore d'une traduction sélective des parties les plus longues entre prose et vers de chacun des chapitres du

21. $C f$. LEE, D.-J., 1981.

22. $C f$. KwON 1993. 
Miaofalianhuajing, accompagnée d'un commentaire plus complet. Deux ans plus tard, en 1461, sur ordre du même roi, le Gan'gyŏng dogam (Institut pour la publication des sūtra) ouvrit ses portes au sein du temple Wŏn'gaksa à Séoul et publia divers sūtra traduits en hunmin jŏngūm. Le MBRHG, la première traduction coréenne intégrale du Miaofalianhuajing, fut ainsi publié en 1463, en tant que deuxième sūtra après le Nūng'ga'gyŏng (skt. Lañkāvatārasūtra), contenant le Miaofalianhuajing yaojie de Jiehuan (?-1129?), moine chinois de l'école Chan de la dynastie des Song (960-1279), et le Fahuajing kezhu 法華經科註 (Commentaire du Sütra de fleur de la loi) d'Yiru 一如, moine chinois de l'école Tiantai de la dynastie des Ming (13681644). Cependant, l'intention de Sejo de favoriser la propagation du bouddhisme ne fut pas reprise par ses successeurs. Par conséquent, le bouddhisme perdit ses privilèges et subit une pression de plus en plus forte tout au long de cette dynastie. Pourtant, malgré l'attitude sévère de la cour envers le samgha, le peuple conserva la croyance bouddhique, ce qui permit au bouddhisme de tisser des liens plus forts avec celui-ci. Durant cette période de persécution, la synthèse définitive des deux courants bouddhiques, le gyojong et le sŏnjong l'école Chŏntae étant alors considérée comme une école du courant sŏnjong - s'acheva sous la direction de Hyujŏng (1520-1604) dans la continuité de l'esprit œcuménique de Wŏnhyo, de Ūichŏn et de Chinul. Cet esprit œecuménique dominera désormais le bouddhisme coréen.

\section{LES PARTICULARITÉS DU MYOB̆̈B RYŎNHHWA'GYŎNG}

Aujourd'hui, nous ne pouvons reconstituer qu'à peine deux exemplaires du MBRHG de l'année 1463. D'où la difficulté d'accès aux sources textuelles et la publication de la version photographique du MBRHG réalisée en 1960 par l'université Dongguk à Séoul: le Sejo wangjo guk'yŏk jang'gyŏng myobŏp yŏnhwa'gyŏng 世祖 王朝國譯藏經妙法蓮華經 (Sūtra du lotus de la loi merveilleuse traduit en coréen par le roi Sejo de Chosŏn) ${ }^{23}$. En nous appuyant sur cette édition contemporaine, nous constatons que le MBRHG

23. Les sept volumes photographiés sont désormais consultables sur le site de la bibliothèque de l'université Dongguk: http://lib.dongguk.edu. 
reprend le même ordre de présentation que le Miaofalianhuajing de Kumārajīva divisé en vingt-huit chapitres et présenté en sept volumes $^{24}$, à l'exception du volume I présenté en trois fascicules séparés. Sur la couverture de chaque volume du MBRHG, le titre de l'ouvrage et les titres des chapitres contenus dans le volume respectif sont écrits en chinois, et non en hunmin jŏng'um. Le texte, qui se lit de droite à gauche et de haut en bas, est imprimé sur le papier traditionnel à partir de planches de bois gravées en relief - la xylographie n'est pas parvenue jusqu'à nos jours. La taille d'une page comprenant neuf lignes d'écriture est de $21,5 \mathrm{~cm}$ par $18 \mathrm{~cm}$. Sur chaque ligne sont insérés soit dix-sept gros caractères chinois, soit trente-quatre petits caractères chinois ou coréen prémoderne. Le fascicule 1 (88 pages) du volume I contient six préfaces ou introductions et la liste des personnes engagées pour cette édition ${ }^{25}$. Le Miaofalianhuajing, le Fahuajing kezhu d'Yiru, la traduction coréenne du Miaofalianhuajing, le Miaofalianhuajing yaojie de Jiehuan et la traduction coréenne de ce dernier sont donnés à partir du fascicule 2 du volume I. Cependant, il ne s'agit pas d'une présentation successive des cinq documents entiers l'un suivant l'autre, mais d'une présentation cyclique de ces cinq documents entrecoupés paragraphe par paragraphe: d'abord, un paragraphe du Miaofalianhuajing écrit en gros, puis le commentaire d'Yiru sur ce paragraphe écrit en petit, suivi de la traduction coréenne du paragraphe du Miaofalianhuajing écrite en petit et du commentaire de Jiehuan sur le paragraphe écrit en gros, enfin la traduction coréenne de ce dernier écrit en petit. Par ailleurs, nous n'avons guère d'informations sur l'identité et le rôle respectif des dix-neuf personnes engagées pour cette édition, à l'exception du nom du directeur d'édition, Sa-Ro Yun, des noms des traducteurs, KyeHee Han et Su-On Kim, et de l'indication selon laquelle le roi Sejo aurait supervisé lui-même cette publication.

24. Le volume I contient les chapitres 1 et 2 ; vol. II, chap. 3 et 4 ; vol. III, chap. de 5 à 7 ; vol. IV, chap. de 8 à 13 ; vol. V, chap. de 14 à 17 ; vol. VI, chap. de 18 à 23 ; vol. VII, chap. de 24 à 28 .

25. Le fascicule $1 \mathrm{du}$ volume I contient la préface du directeur d'édition, Sa-Ro Yun, la liste des dix-neuf personnes engagées, la préface d'un maitre de prince nommé Yo-Gwang $\mathrm{OH}$, celle du moine chinois Yiru, l'introduction du moine chinois Daoxuan, celle du moine chinois Jinan et celle du moine chinois Jiehuan. 


\section{Réception des terminologies bouddhiques chinoises}

En ce qui concerne les particularités linguistiques et doctrinales du MBRHG, nous constatons tout d'abord que le MBRHG a retranscrit en hunmin jŏng'um la majorité des termes techniques bouddhiques chinois du texte de Kumārajīva et du commentaire de Jiehuan, qui ont été eux-mêmes soit translittérés phonétiquement soit traduits sémantiquement à partir de termes sanskrits. Le style de traduction du MBRHG s'avère plus littéral et soigné, sans négliger aucun détail, par rapport au style de la version intégrée dans le Syŏgbo ssangjyŏl (1447), qui est plutôt libéral et plus indigène en raison de l'emploi plus fréquent des mots coréens. L'objectif de la traduction du Syŏgbo ssangjyŏl semble avoir été de transmettre les messages essentiels du Sütra du Lotus, tandis que le MBRHG présente une traduction extrêmement précise. C'est vraisemblablement en raison de ce souci d'exactitude que les éditeurs-traducteurs coréens du MBRHG auraient retranscrit en hunmin jŏng'um les termes bouddhiques chinois. Néanmoins, il existe de rares cas d'emploi de termes spécifiquement coréens à la place de termes bouddhiques chinois. Voici quelques exemples. Le terme bouddhique chinois pusa 菩薩 translittéré phonétiquement à partir du terme sanskrit bodhisattva (être [en marche vers] l'Éveil) est retranscrit en hunmin jŏng'um par bbosal (I.21 $\left.{ }^{26}\right)$, tout comme les termes désignant les quatre membres du samgha: skt. bhikșu (moine mendiant) $>$ chin. biqiu 比丘 $>$ hun. bbiku (I.22); skt. bhikșunī (moniale) > chin. biqiuni 比丘尼 $>$ hun. bbikuni (I.33); skt. upāsaka (laïc pieux) > chin. youposai 優婆塞 $>$ hun. ubbasū (I.59); skt. upāsika (laïque pieuse) > chin. youpoyi 優婆夷 > hun. ubbai (I.59). Dans cette catégorie, d'autres termes techniques bouddhiques importants sont à relever: skt. Śăkya(muni) > chin. Shijia 釋迦 > hun. Syŏgga (I.112); skt. śramana (moine errant) $>$ chin. shamen 沙門 > hun. samon (I.107); skt. nirvāna (extinction) $>$ chin. niepan 涅槃 > hun. nyŏlbban (I.25); skt. dhārañī (formules détentrices) > chin. tuoluoni 陀羅尼 > hun. ddarani (I.37), etc.

Les mots translittérés en hunmin jŏng'um à partir des termes chinois qui traduisent eux-mêmes sémantiquement le sens des substantifs sanskrits sont, quant à eux, plus nombreux: skt. dharma (loi, doctrine) > chin. fa 法 > hun. bŏb (I.20); skt. śrāvaka

26. Les références renvoient au numéro et à la page du volume concerné du MBRHG de l'édition photographique de l'année 1960. 
(auditeur) > chin. shengwen 聲聞 > hun. syŏngmun (I.21); skt. kleśa (passion) > chin. fannao 煩惱 > hun. bbŏnno (I.22); skt. avidyā (ignorance) $>$ chin. wuming 無明 > hun. mu'myŏng (I.24); skt. jñana (connaissance) $>$ chin. $z h i$ 智 $>$ hun. di (I.25); skt. karman (acte) > chin. ye 業 > hun. $\breve{b} b$ (I.26); skt. samādhi (concentration) $>$ chin. ding 定 > hun. dyŏng (I.26); skt. prajña $\bar{a}$ (sagesse) > chin. hui 慧 > hun. hhyuye (I.26); skt. sattva (êtres vivants) > chin. zhongsheng 衆生 > hun. jyungsaing (I.37); skt. upāyakauśalya (expédients salvifiques) $>$ chin. fangbian 方便 $>$ hun. bangbbyŏn (I.38); skt. ekayāna (véhicule unique) > chin. yisheng 一乘 > hun. ilssing (I.43); skt. bhagavat (bienheureux, vénéré du monde) > chin. shizun 世尊 > hun. syejon (I.54), etc.

Par ailleurs, nous pouvons aussi observer l'usage d'une double morphologie d'un même terme, c'est-à-dire soit translittéré phonétiquement soit traduit sémantiquement: skt. arhant (méritant) $>$ chin. aluohan 阿羅漢 > hun. arahan (I.22), ou chin. yinggong 應 供 (digne d'offrande) > hun. ūng'gong (I.25); skt. Śäriputra > chin. Shelifu 舍利弗 > hun. Syaribul (I.30), ou chin. Shenzi 身子 > hun. Sinja (I.32); skt. Subhūti > chin. Xuputi 須菩提 $>$ hun. Syubboddye (I.30), ou chin. Shanji 善吉 > hun. Ssyŏn'gil (I.32), etc. Si Kumārajīva (344-413) employa souvent les termes translittérés phonétiquement, Jiehuan (?-1129?), sept siècles plus tard, utilisa plutôt les termes traduits sémantiquement. Les éditeurs-traducteurs coréens ont donc repris les deux morphologies en les translittérant en hunmin jŏng'um.

Le terme coréen butyŏ 부텨 (I.19), écrit uniquement en hunmin jŏng'um sans aucun caractère chinois ajouté, désigne le Buddha Sākyamuni, tandis que le terme translittéré bbul (I.26), venant du chinois fo(tuo) 佛(陀) et du sanskrit buddha (éveillé), est employé pour désigner les buddha au pluriel. Le terme coréen jyung 즁 (I.82) signifiant le moine est également inséré dans le texte, malgré l'existence du synonyme coréen bbiku issu du chinois biqiu (skt. bhikșu). Les deux termes suivants, maam et hanal, ne sont pas spécifiquement bouddhiques mais occupent une place non négligeable dans le bouddhisme coréen, quand ils sont employés en tant que terminologie bouddhique. Le maam 마암 (I.23) signifiant cœur, pensée ou esprit correspond au terme chinois xin 心 (cor. sim, skt. citta), qui fut souvent employé pour indiquer l'essence de l'être humain ou même la bouddhéité: par exemple, l'ilsim (cœuresprit unique) chez Wŏnhyo (617-686) du royaume Silla Unifié ou 
le chinsim (cœur-esprit véritable) chez Chinul (1158-1210) de la dynastie Koryŏ. Le MBRHG adopte définitivement le terme coréen maam à la place du sino-coréen sim. Le mot d'origine coréenne hanal 하날 (I.56) est choisi pour traduire le terme chinois tian 天 (cor. tyŏn, ciel) traduisant le terme sanskrit deva (dieu), malgré une divergence sémantique subtile entre le hanalltian et le deva. Par ailleurs, tous ces termes coréens avaient déjà été employés dans les deux traductions précédentes du Miafalianhuajing de l'année 1447 et de l'année 1459.

\section{Addenda explicatifs des notions étrangères}

Nous pouvons également considérer comme une particularité stylistique du MBRHG, la mise entre parenthèses d'explications détaillées de la signification de certains termes bouddhiques chinois ou certains mots d'origine chinoise dans la traduction du Miaofalianhuajing et celle du commentaire de Jiehuan. Ceci était entrepris par les éditeurs-traducteurs coréens, vraisemblablement dans le souci de rendre plus accessible la lecture du document. Par exemple, Jiehuan commente les premières phrases du Miaofalianhuajing - «J'ai entendu comme ceci. À un moment donné, le Buddha demeurait dans la cité Maison du roi/Rājagṛha, sur le mont Pic du vautour/Gridhrakūṭa 如是我聞一時 佛住王舍 城者闍堀山中»-de la manière suivante:

«[La phrase] 'J'ai entendu une telle loi du Buddha' est prononcée par Ānanda qui a pris, lors du rassemblement 結集 $^{27}$ [...], la parole le premier sur place, et [cette phrase] prouve juste qu'il y a eu un lieu de transmission de la loi. Il n'est donc pas nécessaire de l'expliquer en détail. L'expression 'à un moment donné' ne désigne pas un moment précis de prédication du Buddha, car elle est utilisée ainsi dans divers sūtra. Le Wangshecheng est la cité située à côté du Mont sacré qui appartient au royaume Magadha, c'est-à-dire le monde de la région de l'Ouest. Le Mont sacré évoqué ici est celui qui ressemble à la tête du vautour. Il a obtenu ce nom en raison de sa forme. Ce fut la demeure du Buddha dans le passé. [...] Chacune des terres où le Buddha a prêché porte [un nom qui a] un sens important. [Le Buddha] se déplaçait, selon le Huayanjing, dans dix lieux pour exposer complètement le monde de la loi. Le Yuanjuejing ${ }^{28}$ s'appuie sur le réceptacle du grand éclat

27. Chin. jieji, skt. samgìti, rassemblement, concile.

28. Chin. Dafangguang yuanjue xiuduoluo leyijing 大方廣圓覺修多羅了義經, skt. Mahāvaipulyapūrnabuddhasūtra, Sūtra de l'Éveil complet. 
lumineux pour montrer directement le cœur-esprit qui éveille la racine et la source. Le présent [sūtra] s'appuie sur le monde humain, en égalisant la souillure et la pureté, pour éclaircir le sens de la fleur du lotus. Le fait de s'appuyer sur les terres du Buddha du passé permet [...] de montrer que la succession de la voie 道 ${ }^{29}$ de la lampe lumineuse continue. Ce sūtra assemble les circonstanciels 權 ${ }^{30}$ pour conduire vers la vérité inchangeable 實 $^{31}$. Adapté aux facultés des auditeurs, il est exposé pour la première fois. Les boddhisattva sont la multitude principale, et les humains et le ciel sont la multitude protectrice externe.» (I.20)

Jiehuan propose ici plutôt une explication succincte sur le contexte du concile de Rājagṛha en faisant un lien avec les lieux de prédication du Buddha selon les trois sūtra. Les éditeurs-traducteurs coréens expliquent, quant à eux, que le terme chinois jieji veut dire «rassembler, c'est-à-dire rassembler les enseignements du Buddha afin d'en faire des sūtra» (I.20).

Après le passage du Miaofalianhuajing sur les arhant - «Ayant obtenu leur bien propre et mis fin aux attachements 結 $32[\ldots]$ de toute existence, ils avaient obtenu la liberté d'esprit $»^{33}-$, les éditeurs-traducteurs coréens ajoutent une explication sur neuf sortes de jie (avidité, colère, arrogance, ignorance, vision, possession, doute, jalousie, attachement), et soulignent que «ces neuf choses s'incorporent dans les passions, en se consolidant mutuellement, et qu'elles deviennent difficiles à détruire; c'est la raison pour laquelle elles sont appelées gyŏl, qui veut dire faire nœuds» (I.23).

La définition du terme anouduoluo sanmiaosan puti 阿礳多羅 三䅐三菩提 (hun. anogdara sammyagsam bboddye, skt. anuttara samyaksaṃbodhi) est donnée par les éditeurs-traducteurs coréens et non par Jiehuan: cela signifie «l'Éveil complet et parfait sans supérieur» (I.37). Ce sont également les éditeurs-traducteurs coréens qui fournissent une explication de la notion de bashi (hun. balsig, huit consciences), notion importante dans l'école mahāyānique indienne Vijñānavāda-Yogācāra, puis dans l'école chinoise Faxiang:

«Les huit consciences sont composées de six consciences, de

la septième conscience malnasig ${ }^{34}$ et de la huitième conscience

29. Chin. dao, voie, chemin amenant à l'Éveil, Éveil.

30. Chin. quan, circonstanciel, autre appellation de fangbian (skt. upāyakauśalya).

31. Chin. shi, vérité éternelle et absolue.

32. Chin. jie, hun. gyŏl, skt. bandhana, nœud, entrave, attachement.

33. Cf. Robert 1997a, p. 47; Burnouf 1852, p. 1.

34. Chin. monashi 末那識, skt. klisțamanas, conscience souillée. 
$\operatorname{araeyasig}^{35}$. L'œil, l'oreille, le nez, la langue, le corps et la pensée sont les six facultés. La couleur, le son, l'odeur, le goût, le toucher et la loi sont les six souillures: ce que les yeux voient est la souillure de la couleur; ce que les oreilles entendent est la souillure du son; ce que le nez sent est la souillure de l'odeur; ce que la langue goûte est la souillure du goût; ce qui est sale au corps est la souillure du toucher; l'attachement au sens, c'est la souillure de la loi. Ce sont ensemble douze lieux. Les six consciences sont originellement issues d'un cœuresprit, mais ce seul cœur-esprit, en s'appuyant sur les six facultés, devient les six consciences [...] L'araeya veut dire semence et aussi matrice de conscience, car elle contient la semence de toutes les choses de l'au-delà du monde et celle du monde. Le malna veut dire intention; comme elle pense constamment au détail, elle est appelée intention. Sur la base de la huitième naît l'idée du Soi; cette pensée n'est que dans la septième, et elle n'est pas dans les autres consciences; c'est pourquoi elle seule est appelée intention. Les six premières consciences sont nommées ainsi selon la faculté, et la septième est nommée ainsi selon la quintessence.» (I.101s)

Quant à la notion de wushibajiao (huit doctrines en cinq périodes) de l'école Tiantai ou de Zhiyi, elle est décrite par les éditeurstraducteurs coréens dans deux passages différents: les cinq périodes de prédication du Buddha ( $c f$. II.5); les huit doctrines ( $c f$. III.4345). En outre, la précision des éditeurs-traducteurs coréens sur le sens des notions rudimentaires, telles que l'octuple chemin juste ( $c f$. II.92) et la posture du lotus ( $c f$. I.55), fait supposer un cercle de lectorat large constitué de ceux qui n'avaient pas suffisamment de connaissance ni sur la doctrine ni sur la pratique bouddhique.

\section{Tendances syncrétique et œcuménique}

Les trois autres particularités suivantes rencontrées dans le MBRHG sont liées aux spécificités doctrinales du Miaofalianhuajing yaojie de Jiehuan: le syncrétisme extracommunautaire, l'œcuménisme intracommunautaire et l'illustration des caractéristiques de l'école Chan. La tendance syncrétique ou «symbiotique» de Jiehuan à l'égard du confucianisme et du taoïsme se manifeste aisément dans son commentaire qui compulsa et cita des livres canoniques confucéens et taoïques afin de démontrer les possibles similitudes entre certaines notions appartenant aux trois courants de pensée. Nous rappelons que le commentaire de Jiehuan

35. Chin. alaiyeshi 阿賴耶識, skt. ālayavijñāna, conscience de tréfonds, conscience-réceptacle. 
date de l'époque des Song (960-1279), durant laquelle la recherche des éléments communs entre le confucianisme, le bouddhisme et le taoïsme s'était généralisée. Par exemple, en commentant le premier paragraphe du Miaofalianhuajing, Jiehuan emploie un terme chinois chargé d'histoire, le dao 道 ( $c f$. I.21), pour traduire le terme sanskrit bodhi (éveil). Il est connu que le dao désigne aussi bien l'ordre cosmique confucéen que le principe suprême taoïque, et que ce terme ait été adopté par les bouddhistes chinois de la première génération pour traduire le terme sanskrit dharma ${ }^{36}$, puis plus tard pour désigner la bodhi. Les termes quan et shi renvoyant respectivement aux moyens provisoires et aux méthodes générales et permanentes issus du Mengzi, un des Quatre Livres confucéens, sont employés pour expliquer la notion de fangbian (skt. upāyakauśalya), une des principales doctrines du Sütra du Lotus ${ }^{37}$. En expliquant les vertus des dix grands disciples du Buddha, Jiehuan fait également allusion aux dix sages disciples de Confucius ( $c f$. I.32). En commentant le passage du Miaofalianhuajing sur le parinirvāṇa du Buddha, Jiehuan semble chercher à démontrer par la citation d'un érudit confucéen et du Laozi, livre canonique taoïque ( $c f$. I.109), la similitude des états de constance selon les trois courants de pensée. En évoquant le Chunqiu (Annales des printemps et automnes), un des Cinq Classiques confucéens, Jiehuan constate que l'attitude respectable, qui consiste à s'agenouiller devant un être honorable, est commune aussi bien en Inde qu'en Chine ( $c f$. II.178). Jiehuan compare également les huit trigrammes, exposés dans le Yijing (Classique des mutations), ouvrage antique de divination et un des Cinq Classiques confucéens, avec les huit directions bouddhiques (cf. III.156s).

Ce syncrétisme extracommunautaire de Jiehuan est confirmé par les éditeurs-traducteurs coréens qui ajoutèrent, dans le MBRHG, leurs propres précisions commentées. Par exemple, au sujet de la comparaison faite par Jiehuan entre les dix grands disciples du Buddha et les dix sages disciples de Confucius, ils poursuivent la réflexion en écrivant que chacun d'entre eux était doué d'une grande vertu ( $c f$. I.32). Lorsqu'ils en viennent à expliquer la notion de yingxiangzhong (influençant), les éditeurs-traducteurs coréens

36. Cf. MalmQuist 1968/1972, p. 40; Demiéville 1973a, p. 23.

37. Cf. Kıм, J.-H., 2006, p. 134-139; Kıм, Y.-S., 2009, p. 62. 
font allusion à la notion taoïque de wuwei ${ }^{38}$ (non-agir) : «Tous les buddha et les bodhisattva du corps de la loi du passé [...] aidaient le roi de la loi, comme si les étoiles encerclaient la lune, et même s'ils ne faisaient rien, ils étaient très utiles » (I.26). Ce sont aussi les éditeurs-traducteurs coréens qui décrivent les personnages Gushe et Aitaita, qu'évoque Jiehuan ( $c f$. II.28), et qui sont en effet mentionnés dans le Zhuangzi, livre canonique du taoïsme. Ils explicitent même le terme chugou (chien de paille), également issu du Zhuangzi, avec lequel Jiehuan compare les deux véhicules ( $c f$. II.212), c'est-à-dire le petit véhicule (skt. hīnayāna). Enfin, les éditeurs-traducteurs coréens citent eux-mêmes le Zhuangzi pour expliquer le sens du terme $x u$ (vide) en le comparant avec le fait de vouloir décrire, en vain, la grandeur d'océan à une grenouille enfermée dans un puits, dont la vision est fort limitée (cf. III.156).

Parallèlement à ce syncrétisme extracommunautaire, nous pouvons constater une tendance œcuménique intracommunautaire bouddhique chez Jiehuan. Ce dernier essaie en effet de démontrer les correspondances doctrinales entre le Huayanjing et le Fahuajing, et d'intégrer des enseignements de diverses écoles bouddhiques chinoises, notamment Tiantai et Faxiang. Cet œcuménisme intracommunautaire fut par ailleurs une des caractéristiques du bouddhisme chinois durant des siècles après la grande proscription de l'année 845. En citant, tout au long de son commentaire, plus d'une quarantaine de passages issus de divers sūtra ${ }^{39}$, Jiehuan complète ses propos, sans les critiquer, et insiste surtout que le Huayanjing et le Fahuajing enseigneraient la même doctrine ( $c f$. I.14), bien qu'il y ait une différence de période d'enseignement due à la diversification des facultés des auditeurs. Quant aux enseignements de diverses écoles bouddhiques, celui de Zhiyi de l'école Tiantai est le plus souvent intégré et évoqué. Par exemple, Jiehuan explique, en se référant au Miaofalianhuajing wenju (Explication textuelle du Sütra du lotus de la loi merveilleuse, T 1718, vol. 34) de Zhiyi, les trois

38. Cf. Le Daodejing, chapitres 2, 3, 10, 29, 37, 38, 48, 57, 63; Julien 1996.

39. Notamment, le Huayanjing (III.17, 167; IV.16, 61; V.89; VI.44, 101, 118, 137, 177; VII.5, 156, 157, 161), le Lengyanjing ou Śüramgamasūtra (V.165; VI.29, 59; VII.51, 69, 103, 106, 159, 173), le Weimojiejing ou Vimalakītinirdeśasūtra (I, 216; III.55; IV.12, 49, 126); le Yuanjuejing (II.74; VII.5, 173), le Daborejing ou Mahāprajñāpāramitāsūtra (I.216; II.218), le Dajijing ou Mahāsannipātasūtra (VI.174), etc. 
sens du terme sanskrit arhant: digne personnage; homme parvenu au degré le plus élevé parmi les bhikṣu; meurtrier ou vainqueur de l'ennemi (cf. I.25, 79) ${ }^{40}$. En commentant la notion des dix ainsités ${ }^{41}$, Jiehuan reprend l'idée de Zhiyi - ou de Huiwen ( $\mathrm{VI}^{\mathrm{e}}$ siècle) considéré comme le premier patriarche de l'école Tiantai - sur les trois visions ou vérités ${ }^{42}$, mais sans aucune explication détaillée:

«Les aspects réels évoqués ci-dessus sont la nature, l'aspect, la substance, la force et la cohérence du début à la fin des entités. Ce qui est visible, c'est l'aspect. La racine et le fondement de l'aspect, c'est la nature. Ce qui porte une forme, c'est la substance. Ce qui correspond à l'usage bénéfique, c'est la force. Ce qui se passe momentanément, c'est l'action. Ce qui engage, c'est la cause. Ce qui assiste la cause, c'est la condition. Le fruit de la condition, c'est l'effet. Ce qui répond à l'effet, c'est la rétribution. Le benmo veut dire début et fin. Le jiujing veut dire atteindre. Les entités ne peuvent pas quitter ces dix ainsités 如 ${ }^{43}$ et elles sont munies de ces dix. L'expression 'c'est ainsi' désigne la loi, comme il est dit: les entités ont l'aspect qui est ainsi, la nature qui est ainsi, le début et la fin qui sont ainsi, et l'épuisement qui est ainsi; ce sont tous des aspects réels. [...] Partant seule de la vérité séculière, tout n'est autre que l'aspect réel. Donc, celui qui veut l'attester, ne détruira pas la vérité céleste, ni ne mettra la présente substance à terme; il l'obtiendra paisiblement en dehors du cœur-esprit de la forme. La loi primordiale est difficile à comprendre. Jadis, on parlait de quatre saints, six ordinaires et dix mondes de la loi; chaque monde est muni de dix ainsités, donc dix mondes avec cent ainsités, et en somme cent mondes avec mille ainsités. S'ils se fusionnent, ils deviennent infinis. Cela montre la nature de la loi dans les aspects réels. On a encore établi trois visions et on les a expliquées mutuellement: [selon la vision de] vacuité 空 ${ }^{44}$, l'aspect est identique; [selon la vision de] virtuel 假 ${ }^{45}$, l'ainsité est apparente; [selon la vision de] milieu 中 ${ }^{46}$, l'aspect est ainsi. C'est pour éclairer la sagesse de discernement dans l'aspect réel. [...].» (I.148s)

Concernant le passage du Miaofalianhuajing sur les huit fils du dernier Buddha nommé Riyue dengming ${ }^{47}$ (skt. Candrasūryapradīpa,

40. $C f$. Zhiуı, Le Miaofalianhuajing wenju (Explication textuelle du Sūtra du lotus de la loi merveilleuse), T 1718, vol. 34, p. 7 ; KIM, Y.-S., 2009, p. 22.

41. $C f$. Robert 1997b: cette liste de «dix» ainsités ne se trouve pas dans le texte népalais traduit par E. Burnouf, ni dans la traduction de Dharmarakșa.

42. Cf. Petzold 1987, p. 35-39; Cha 2010, p. 48s, 54.

43. Chin. $r u$ : ainsité inchangeable, permanente.

44. Chin. kong, vacuité. Selon cette vision, les entités sont vides; elles n'ont pas de substance, ni la nature propre.

45. Chin. jia, virtuel. Selon cette vision, les entités vides et irréelles ont une apparence, mais une virtuelle.

46. Chin. zhong, milieu. Ce serait une vision de percevoir les entités, sans extrémité ni illusion.

47. $C f$. Robert 1997a, p. 58s; Burnouf 1852, p. 11s. 
lampe lumineuse du soleil et de la lune), Jiehuan essaie d'établir un lien entre ces huit princes et les huit consciences selon le Vijñānavāda-Yogācāra ou le Faxiang:

«Cela montre les circonstances de transmission et de préservation de la loi merveilleuse en évoquant le début de Randeng 然燈48. Entre le premier Dengming et le dernier Dengming, il y a vingt mille buddha; Randeng est le dernier prince Dengming. Les prénoms et les noms de famille de chacun étaient identiques; c'est pour montrer que leur principe de l'Éveil était identique. Il y avait huit princes; c'est pour exprimer la loi en retraçant les vestiges du saint. Dengming avait huit intentions; cela montre qu'il y a l'intention qui observe subtilement sur la base du cœur-esprit merveilleusement lumineux et vrai, dont l'utilité est de nombre huit. Ce cœur-esprit merveilleux est vide à l'origine mais il est utile; c'est pourquoi son nom est Muni-d'Intention; c'est l'existence merveilleuse. En émergeant du cœur-esprit merveilleux, il n'a aucune mauvaise utilité; c'est pourquoi son nom est BonneIntention; c'est le bien merveilleux. Sa quantité ne pouvant pas être mesurée, son nom est Intention-Incommensurable; c'est la quantité merveilleuse. En face d'objet, il est utile; c'est pourquoi son nom est Intention-Précieuse; c'est le trésor merveilleux. En contact avec la foule, il se prolonge; c'est pourquoi son nom est Intention-Accrue; c'est l'ajout merveilleux [...]. En ayant de bonnes connaissances, son nom est Intention-Indubitable [...] ; c'est l'Éveil merveilleux. Il répond aux choses comme l'écho résonne, c'est pourquoi son nom est Intention-Résonante; c'est la résonance merveilleuse. Il établit dix mille lois, c'est pourquoi son nom est Intention-de-Loi; c'est la loi merveilleuse. [Les huit princes] gouvernaient les quatre continents lorsqu'ils n'avaient pas quitté la famille; cela veut dire qu'ils étaient encore attachés à des choses. En entendant que leur père avait obtenu l'Éveil, ils ont renoncé au trône et ont quitté la famille; cela montre qu'il faut oublier l'intention et éliminer l'attachement pour atteindre l'Éveil juste. Le prince Victoire-de-Sagesse, qui jouait avec des jouets précieux, abandonne ceux-ci et part, en entendant que son père avait obtenu l'Éveil ; comme lorsque le roi du cœur-esprit quitte la maison, le triple monde, les fils huit consciences le suivent et deviennent maîtres de la loi.» (I.100s)

\section{Primauté de l'herméneutique chan/sǒn}

Une dernière des particularités du MBRHG concernerait l'appropriation de l'épistémologie et de l'herméneutique de la réalitévérité telles qu'elles sont perçues par le commentateur. Parallèlement à son effort à la fois d'un œcuménisme intercommunautaire et d'un syncrétisme interreligieux, Jiehuan interprète le Miaofalianhuajing

48. Skt. dīpamkara ou dīpamahara, lumière naturelle. 
en tant que moine Chan, dont le point de vue semble s'être accordé aux attentes des éditeurs coréens du MBRHG. Prenons quelques exemples d'exposition de cette perception. Dès le début de son commentaire sur le premier paragraphe du Miaofalianhuajing, Jiehuan se montre fidèle à la pensée de la lignée Chan : «C'est pour montrer directement le cœur-esprit qui éveille la racine et la source. Le présent [sūtra] s'appuie sur le monde humain, en égalisant la souillure et la pureté » (I.21). Si la première phrase de Jiehuan nous rappelle une des quatre strophes, rassemblées probablement durant la période des Tang (618-907) afin de résumer la quintessence du Chan, «Pointer directement au cœur-esprit de l'homme 直指人 心 » ${ }^{49}$, sa deuxième phrase fait allusion à l' aspect trans-dichotomique entre la vie mondaine et l'état d'Éveil, qu'illustrent par ailleurs divers sūtra mahāyāniques et des moines Chan chinois ${ }^{50}$. Ainsi, Jiehuan affirme que la vraie extinction (skt. nirvāṇa) serait au-delà de la vacuité (skt. śūnyatā) et de la forme (skt. rūpa) (cf. II.23).

Reconnaître tout être vivant comme buddha potentiel (skt. tathāgatagarbha, embryon de l'Ainsi-venu/allé), muni de l'Éveil originel ou de la sagesse originelle, est également un autre aspect primordial prôné, au moine au niveau initial, par l'école $\mathrm{Chan}^{51}$, et Jiehuan le confirme à plusieurs reprises: les êtres vivants, ne connaissant ni la lumière originelle ni la sagesse originelle, seraient prisonniers d'eux-mêmes et auraient du mal à atteindre le corps merveilleux ( $c f$. I.61; III.85); si la clarté originelle des êtres s'expose et si la sagesse originelle apparait, cette apparition éclairée et complète ne serait autre que celle du Buddha ( $c f$. I.64); les êtres vivants seraient originellement munis de la nature de buddha $(c f$.

49. $C f$. Tianxi Guo, la préface du Linjihuizhao chanshi yulu (Recueil de paroles de l'illustre maître chan Linji), T 1985, vol. 47, p. 495.

50. $C f$. Le Borexinjing (skt. Prajñāpāramitāhrdayasūtra; Sūtra du cour de la perfection de sagesse), T 251, vol. 8, p. 848 : «La forme n'est pas différente de vacuité, et la vacuité n'est pas différente de forme; la forme est vide, et la vacuité est la forme 色不異空 空不異色 色郎是空 空郎是色》; le Jingangjing (Sūtra du diamant), T 235, vol. 8, p. 749 : «Bien que d'innombrables êtres vivants aient ainsi atteint le nirvāṇa, il n'y a en réalité aucun être vivant qui a atteint le nirvāṇa 如是 滅度無量無數無邊衆生 實無衆生得滅度者》; SENGCAN, Le Xinxin ming (Inscription sur la foi en cour-esprit), T 2010, vol. 48, p. 377 : «Dans le monde de la loi de la vraie ainsité, il n'y a ni autrui ni soi [...] L'existence est la non-existence; la nonexistence est l'existence 眞如法界 無他無自 [...]有即是無 無即是有》.

51. Cf. Yonguia, Le, Zhengdao ge (Chant de la réalisation de la Voie), $\mathrm{T} 2014$, vol. 48, p. 395 : «La vraie nature de l'ignorance est la nature de buddha [...]; notre nature est le vrai buddha originel 無明實性即佛性 [...] 本源自性天眞佛》. 
II.162; VI.78); de ce fait, le saint et le commun ne seraient pas différents ( $c f$. II.160; IV.179).

La phrase du Miaofalianhuajing, «leur esprit n'est rattaché à rien $»^{52}$ signifierait selon Jiehuan le fait de «rechercher l'Éveil en s'éloignant de l'enseignement doctrinal et en oubliant les traces du cœur-esprit» (I.84). Cette interprétation semble renvoyer à une autre strophe de la quintessence du Chan, «Transmission au-delà de l'étude scolastique 呚外別傳》, mais aussi à une expression connue dans la tradition Chan: «Le lieu de l'activité mentale est détruit 心 行處滅 ${ }^{53}$. La raison pour l'éloignement, voire la transcendance des doctrines spéculatives dans la recherche de l'Éveil se trouve dans les passages suivants du commentaire: «La parole ne peut pas atteindre la loi primordiale [...], la loi merveilleuse de l'aspect réel; il est donc inutile d'en parler davantage; la pensée ne pourra pas l'atteindre» (I.145); «l'essentiel de la loi merveilleuse [...], le savoir et la vision de buddha et cette loi prêchée [...], ne sont pas à connaître ni par la pensée, ni par la spéculation, ni par le discernement» (I.174). Ainsi, résonnent-elles aisément l'assertion «Indépendant des textes et des mots 不立文字», une autre strophe de la quintessence du Chan, et l'expression «La voie de la parole est interrompue 言語道斷 ${ }^{54}$.

Sur la voie de l'Éveil, l'autonomie de l'homme est primordiale, et ceci surtout pour un disciple Chan ${ }^{55}$, comme le souligne Jiehuan: «Réaliser l'Éveil de buddha spontanément, cela veut dire qu'on réalise le corps de la sagesse sans recourir aux autres»(I.118). Pourtant, la voie de l'Éveil peut être hétérogène selon les facultés de chacun des pratiquants. En évoquant que l'on peut acquérir l'Éveil de buddha par une simple audition d'une stance issue de texte bouddhique, puisque la bouddhéité serait innée dans la condition humaine, telle une perle mystérieuse cachée dans un

52. $C f$. Robert 1997a, p. 56; Burnouf 1852, p. 9.

53. Cf. Le Dabore boluomiduo jing (skt. Mahāprajñāpāramitāsūtra), T 220, vol. 7, p. 948 : «La nature est loin des mots et de la parole; le lieu de l'activité mentale est détruit 性離文言 心行處滅》.

54. Cf. Le Dabore boluomiduo jing, T 220, vol. 7, p. 422 : «La voie des noms et de la parole est toute interrompue 一切名字言語道斷》; BoJo CHINUL 1991, p. 122, 228; cas ${ }^{\circ} 32$ du Biyanlu (Recueil de la falaise verte), T 2003, vol. 48, p. 171 : Linji aurait donné une gifle à celui qui lui demanda sur le sens du dharma du Buddha.

55. Cf. Cas n ${ }^{\circ} 57$ du Biyanlu, T 2003, vol. 48, p. 191 : «Je suis seul vénérable au-dessus du ciel et en dessous du ciel 天上天下唯我獨尊》. 
vêtement ( $c f$. I.203), Jiehuan semble rappeler, outre la notion de tathāgatagarbha, l'anecdote du sixième patriarche de l'école Chan, Huineng, qui aurait obtenu subitement l'Éveil en entendant une stance du Jingangjing (Sütra du diamant) récité par un voyageur ${ }^{56}$. De même, Jiehuan indique que la vraie extinction ne s'acquiert pas obligatoirement par la pratique ( $c f$. I.212), et rejoint en cela la pensée de certains tenants du Chan ${ }^{57}$ : «Même si on ne la cherche pas, on l'acquiert par soi-même» (I.203). Car, comme l'explique Jiehuan en mettant en avant l'aspect non-dualiste, non-distinctif, «En vérité, l'Éveil n'est pas une chose difficile ou facile $»^{58}$ (I.223). Pourtant, il reconnaît également, dans son commentaire sur le passage du Miaofalianhuajing soulignant l'importance de la foi, que l'on peut commencer ce chemin vers l'Éveil par la foi (I.153). Cette voie de la foi pourrait bien renvoyer à l'une des trois méthodes vers l'Éveil proposées par le maître coréen Chinul (1158-1210): le wŏndon sinhaemun (porte de foi et compréhension complète et subite) pour ceux qui sont munis de facultés moyennes ${ }^{59}$. Cette analogie méthodologique aurait pu être remarquée et même appréciée par les éditeurs du MBRHG et des lecteurs coréens.

Enfin, il est à noter que Jiehuan n'exerce aucune critique à l'égard de certaines pratiques exposées dans le Sütra du Lotus, telles que la pratique de dons, l'offrande aux reliques, la construction de pagodes, l'édification des images et de statues du Buddha, etc. $(c f . \text { I.85, 215-224 })^{60}$. Celles-ci auraient en effet pu faire l'objet de mépris de la part d'un moine Chan iconoclaste ${ }^{61}$. De même, Jiehuan

56. Cf. Le Liuzudashi fabao tanjing (Sūtra de la plateforme du sixième patriarche), T 2008, vol. 48, p. 348.

57. $C f$. Le Jingde zhuandeng lu (Annales de la transmission de la lumière de l'ère Jingde), T 2076, vol. 51, p. 240 : lorsque Mazu s'adonna à la pratique de la méditation assise (chin. zuochan, jap. zazen), son maître Nanyue l'aurait comparé avec le fait de polir une brique pour en faire un miroir.

58. $C f$. SengCan, Le Xinxin ming (Inscription sur la foi en cour-esprit), T 2010, vol. 48, p. 376 : «L'essence de la grande voie est large; elle n'est ni facile ni difficile 大道體宽 無易無難》.

59. $C f$. Bojo Chinul 1991, p. 284, 453s ; Kıм, K.-K., 2007, p. 29s, 62-64.

60. $C f$. Robert 1997a, p. 83-85.

61. $C f$. Cas $\mathrm{n}^{\circ} 1$ du Biyanlu, T 2003, vol. 48, p. 140 : Bodhidharma répond à l'empereur $\mathrm{Wu}$ des Liang que ce dernier ne mérite rien malgré ses bonnes actions en faveur du bouddhisme; le Linjihuizhao chanshi yulu (Recueil de paroles de l'illustre maître chan Linji), T 1985, vol. 47, p. 500 : «Si vous rencontrez le Buddha, tuez le Buddha; si vous rencontrez un patriarche, tuez le patriarche 逢佛殺佛 逢祖殺祖》. 
ne fait aucune mention négative sur la description bouddhique du monde (skt. lokaprajñapti, pāl. lokapaññatti). Au contraire, il l'explique en détail ( $c f$. I.46) et, à sa suite, les éditeurs-traducteurs coréens y ajoutent encore des précisions, comme s'ils acceptaient incontestablement cette cosmologie bouddhique. Une vision de type de «Ni cœur-esprit ni Buddha 非心非佛 ${ }^{62}$ d'un Mazu ou de «Tuez le Buddha, tuez le patriarche 殺佛殺祖» d'un Linji, ne semble plus être d'actualité6 ${ }^{63}$. Tout aussi inattendu que surprenant, une sévère critique fut adressée de la part de Jiehuan aux tenants du xiaosheng (petit véhicule) qui, à son époque, demeurait fort minoritaire ou quasi inexistant en Chine. Cette attaque repose sur l'affirmation, selon laquelle les adeptes du xiaosheng ne pourraient obtenir complètement la nature originelle, ni le nirvāna sans reste, car ils seraient tombés dans l'erreur en cherchant l'Éveil à l'extérieur du Soi (cf. I.109, 145, 177, 203).

\section{Pour conclure}

Le Myobŏb ryŏnhhwa'gyŏng (= MBRHG) de l'année 1463 contenant la première traduction coréenne intégrale du célèbre Miaofalianhuajing (Sūtra du lotus de la loi merveilleuse) de Kumārajīva (344-413) et celle du Miaofalianhuajing yaojie (Compendium du Sūtra du lotus de la loi merveilleuse) du moine Chan chinois Jiehuan (?-1129?) demeure inaperçu en dehors de la péninsule coréenne, malgré son importance au sein du saṃha coréen durant la dynastie Chosŏn (1392-1910), tout comme, par ailleurs, le bouddhisme coréen et son histoire restent un domaine à explorer pour le monde savant français. La présente étude a essayé de présenter quelques aspects de ce document inédit par une observation de son contexte historique, d'une part, et d'autre part, par une analyse de ses particularités linguistiques et doctrinales. Cette double approche nous a permis d'identifier quelques facteurs importants qui ont été à la base de sa réalisation.

62. Cf. Le Jingde zhuandeng lu, T 2076, vol. 51, p. 253.

63. Cf. Cas $\mathrm{n}^{\circ} 37 \mathrm{du}$ Biyanlu, T 2003, vol. 48, p. 175 : «Le triple monde n'existant pas, où veux-tu trouver le cœur-esprit? 三界無法 何處求心》; BoJo Chinul 1991, p. 64: «Tous les buddha du triple monde ne dépendent que du cœur-esprit». 
Le document a été conçu au début de la dynastie coréenne Chosŏn qui avait adopté le néoconfucianisme comme principe de gouvernement. Le roi bouddhiste Sejo, qui régna durant une douzaine d'années (1456-1468), semble avoir cherché à revitaliser le bouddhisme afin de consolider son pouvoir politique. En comptant sur le soutien du peuple bouddhiste, il aurait tenté d'affaiblir l'influence politique de ses opposants, les lettrés confucéens. Ainsi, Sejo supervisa lui-même la traduction et la publication d'un grand nombre de textes bouddhiques, afin de rendre plus accessibles la biographie du Buddha et la doctrine bouddhique au peuple bouddhiste, qui n'avait jusqu'alors ni connaissance suffisante, ni accès direct aux textes bouddhiques rédigés en chinois, la langue savante de l'époque. D'où l'utilisation du hunmin jŏng'ūm, l'écriture coréenne prémoderne promulguée en 1446, pour la traduction de textes bouddhiques. Dans un tel contexte politico-historique, le MBRHG de l'année 1463 a vu le jour.

Sur le plan linguistique, le MBRHG ne se présente pas comme une traduction entièrement «indigène», car la majorité des termes techniques bouddhiques chinois employés dans le Miaofalianhuajing et dans le Miaofalianhuajing yaojie a fait l'objet d'une translittération phonétique en hunmin jŏng'ūm et non d'une traduction sémantique. Ceci est valable pour tous les types de termes bouddhiques, des toponymes aux appellations honorifiques, en passant part les noms propres, les termes doctrinaux et cosmologiques. L'emploi de termes coréens est presque absent, sauf quelques rares cas tels que le butyŏ (Buddha), le jyung (moine), le maam (cœur, esprit) et le hanal (ciel). De ce fait, nous comprenons mieux la raison pour laquelle les éditeurs-traducteurs coréens ont été dans l'obligation d'ajouter, pour les lecteurs bouddhistes coréens non érudits, des explications sur le sens de certains termes techniques bouddhiques chinois. Constatant la seule différence phonétique entre les termes bouddhiques chinois et coréens, nous pouvons alors difficilement en déduire, que les éditeurs-traducteurs coréens auraient fourni autant de perfectionnement linguistique, au moins en ce qui concerne la terminologie bouddhique, que les bouddhistes chinois lorsque ceux-ci, dix siècles auparavant, en vinrent à traduire les sources sanskrites dans leur propre langue, et cela malgré leur tentative de réaliser une traduction exacte et soignée et leur essai d'indigénisation du bouddhisme pour le peuple coréen. 
D'un point de vue doctrinal, il est notoire que seul le commentaire de Jiehuan ait été sélectionné parmi de nombreux commentaires du Sütra du Lotus de l'époque pour être pleinement intégré dans le MBRHG et traduit en coréen. Il s'agit là du choix délibéré d'un commentaire non orthodoxe, peu (re)connu en Chine et au Japon, dont l'auteur appartenait à l'école Chan et non à école Tiantai chinoise ou Chŏntae coréenne. Ce commentaire est devenu, cependant, le commentaire par excellence du Sūtra du Lotus durant la dynastie Chosŏn. Quelles ont pu être les raisons d'un tel choix et de son succès auprès des lecteurs bouddhistes coréens? D'après notre analyse, le commentaire de Jiehuan contient des éléments spécifiques, qui auraient pu correspondre aux attentes des bouddhistes coréens du $\mathrm{Xv}^{\mathrm{e}}$ siècle, notamment un syncrétisme extracommunautaire à l'égard du confucianisme et du taoïsme, un œcuménisme intracommunautaire avec la perception identitaire de l'enseignement du Hwaŏmgyŏng/Huayanjing et de celui du Bŏphwa'gyŏng/Fahuajing et une anthropologie du Sŏn/ Chan reconnaissant en tout homme le tathāgatagarbha (embryon de l'Ainsi-venu/allé). Nous avons pu également constater que les éditeurs-traducteurs coréens se sont inscrits, par l'intermédiaire de leur précision insérée dans le commentaire du moine Chan chinois, dans la même ligne de pensée de ce dernier.

Nous pouvons ainsi conclure que ce qui est spécifiquement coréen dans le MBRHG ou ce qui fait la valeur historique et religieuse du MBRHG ne consiste pas uniquement en une traduction des textes bouddhiques chinois en hunmin jŏng'ūm, mais plus en le choix du commentaire du moine Chan chinois, Jiehuan, qui eut pour visée de maintenir un processus d'harmonisation interreligieuse et d'unification ocuménique et un universalisme de la bouddhéité. Alors, le Sütra du Lotus ou Miaofalianhuajing accompagné du commentaire de Jiehuan demeura durant les siècles suivant la publication du MBRHG et demeure encore aujourd'hui l'un des textes bouddhiques les plus importants et les plus étudiés dans la péninsule coréenne.

kk.kim@unistra.fr 


\section{Bibliographie}

\section{Textes sources}

妙法蓮華經 一 (Myobŏb ryŏnhhwa'gyŏng il, Sūtra du Lotus de la Loi merveilleuse. Volume I), Séoul 1463 (consultable sur le site de la bibliothèque de l'université Dongguk à Séoul: http://lib.dongguk.edu.).

L'UNIVERsité DONGGUK, 世祖王朝 國譯藏經 妙法蓮華經 (Sejo wangjo guk'yŏk jang'gyŏng myobŏp yŏnhwa'gyŏng, Sūtra du lotus de la loi merveilleuse traduit en coréen par le roi Sejo de Chosŏn), Séoul 1960.

$\mathrm{KIM}$, Yong-Bae (tr.), 역주 법화경언해 권1 (Traduction et commentaire de la traduction du Sūtra de fleur de la loi en hunmin jŏng'ūm. Volume I), Séoul 2000.

\section{Ouvrages cités}

AN, Byong-Hee, 1971, «Gaegan bŏphwa'gyŏng ŏnhae'e daehayŏ (Sur la nouvelle édition du Bŏphwa'gyŏng ŏnhae)», Dongbang hakji 12 (Séoul), p. 235-246.

AN, Byong-Hee, 1998, «Bŏphwa'gyŏng ŏnhae'ūi sŏji (Bibliographie du Bŏphwa'gyŏng ŏnhae)», Sŏji hakbo 22 (Séoul), p. 3-33.

BAREAU, André, 1955a, Les premiers conciles bouddhiques, Paris.

BAREAU, André, 1955b, Les sectes bouddhiques du petit véhicule, Saïgon. BAREAU, André, 1966, «Le bouddhisme indien», in Les religions de l'Inde. III. Bouddhisme, jaïnisme, religions archä̈ques, Paris, p. 1-246.

BAREAU, André, 1970, «Le bouddhisme indien », in Histoire des religions, vol. I, Pléiade, Paris, p. 1146-1215.

Bojo Chinul, 1991, Bojo guksajip (Les ouvres du maître national Bojo), Séoul.

Bronkhorst, Johannes, 2007, Greater Magadha. Studies in the Culture of Early India, Leiden-Boston, p. 15-54.

Burnouf, Eugène, 1852, Le Lotus de la Bonne Loi, Paris.

Buswell, Robert E. jr., 1999, «The Koryŏ Periode», in Y. TAKeuchi (éd.), Buddhist Spirituality. Later China, Korea, Japan, and the Modern World, New York, p. 79-108.

CHA, Cha-Sok, 2010, Dasi ig'nūn bŏphwa'gyŏng (Relecture du Sūtra du Lotus), Séoul.

Conze, Edward, 2002, Le bouddhisme dans son essence et son développement, Paris (éd. orig. 1952). 
Demiéville, Paul, 1970, «Le bouddhisme chinois », in Histoire des religions, vol. I, Pléiade, Paris, p. 1249-1319.

Demiéville, Paul, 1973a, Choix d'études bouddhiques (1929-1970), Leiden.

Demiéville, Paul, 1973b, Choix d'études sinologiques (1921-1970), Leiden.

Duccur, Guillaume, 2011, Initiation au bouddhisme, Paris.

FABRe, André, 2000, Histoire de la Corée, Paris.

Fong, Yeou-Lan, 1952, Précis d'histoire de la philosophie chinoise, Paris. Frank, Bernard, 2000, Amour, colère, couleur. Essais sur le bouddhisme au Japon, Paris.

FujitA, Kotatsu, 1980, «Pure Land Buddhism and the Lotus Sūtra», in Indianisme et bouddhisme, Louvain-la-Neuve, p. 117-130.

Fuss, Michael, 1991, Buddhavacana and Dei verbum, Leiden-New YorkKöln.

Harvey, Peter, 1993, Le bouddhisme. Enseignements, histoire, pratiques, Paris.

HiraKawa, Akira, 1990, A history of Indian Buddhism : from Śākyamuni to early Mahāyāna, Hawaii.

HiraKawa, Akira, 1996, «Daesūng bulgyo’esŏ bon bŏphwa'gyŏng'ūi ui' chi (La place du Sūtra du Lotus dans le bouddhisme mahāyāna)», in C.-S. Cha (tr.), Bŏphwa sasang, Séoul, p. 17-53.

Ho, Hung-Sik, 1978, «Koryŏ jŏn'gi bulgyo'gyewa chŏntaejong'ūi hyŏngsŏng gwajŏng (Le bouddhisme au début de la dynastie Koryŏ et la fondation de l'école Chŏntae)», Hanguk hakbo 11 (Séoul), p. 77-110.

HuANG, Kuo-ching, 2011, «Songdai Jiehuande fahua sixiang 宋代戒環 的法華思想 (A Research on the Thought of Jie-huan's A Concise Commentary on the Lotus Sutra)», Jiedi 20 (Taïwan), p. 75-123.

Jogye Order, 2004, What is Korean Buddhism, Séoul.

Jong, Sung-Sok, 1986, Bŏphwa'gyŏng'ūi se'gye (Le monde du Sūtra du Lotus), Séoul.

Julien, Stanislas (tr.), 1996, Lao-tseu. Tao te king ou Livre de la Voie et de la Vertu, Turin.

Kamata, Shigeo, 1994, Hanguk bulgyosa (Histoire du bouddhisme coréen), H.-S. Shin (tr.), Séoul (5 éd.).

KANG, Sun-A, 1997, «Sŏngdalsaeng sŏ'che'gye bŏphwa'gyŏng gyehwanhae'ūi panbon'e gwanhan yŏn'gu (Étude sur la publication du commentaire du Sūtra du Lotus de Jiehuan selon le système d'écriture de Sŏngdalsaeng)», Gasan hakbo 6 (Séoul), p. 34-85. 
KeEL, Hee-Sung, 1984, Chinul: The Founder of the Korean Son Tradition, Séoul.

Kim, Du-Jin, 1984, «Chegwan'ūi chŏntae sasang (La pensée de Chegwan sur la Terrasse céleste)», Hanguk’hak nonchong 6 (Séoul) p. 39-68.

KIM, Hai-Jin, 1958, Buddhism and Korean Culture, New Delhi.

Kıм, In-Taek, 1987, Myobŏp yŏnhwa'gyŏng yŏkju (Traduction et commentaire du Sūtra du Lotus), Chŏngju.

KIM, Jong-Hee, 2006, «Tiantai Zhiyi'ūi wŏndon jigwan'esŏ bangpyŏn'ūi ūimi (Le sens de moyens habiles selon la méthode de concentration et contemplation complète et subite de Zhiyi)», Bulgyohak yŏn'gu 13 (Séoul), p. 134-154.

Kim, Kee-Hyog et NaKamura, Emiko, 2010, Hangūl bŏphwa'gyŏnggwa kana bŏphwa'gyŏng'üi ŏnŏ (La langue du Sūtra du Lotus en coréen et celle du Sūtra du Lotus en japonais), Séoul.

KIM, Kyong-Kon, 2007, Der Mensch und seine Erlösung nach Sŏn-Buddhismus und Christentum. Bojo Chinul und Karl Rahner im Vergleich, Bonn.

KIM, Un-Tae, 1981, «Sejongjo jŏngchi munhwa (La culture politique sous le règne du roi Sejong)», Haksulwŏn nonmunjip 20 (Séoul), p. 113-162.

KıM, Yong-Gil, 1997, «Bŏphwa'gyŏng' ūi ŏnhaewa gū bae'gyŏng (La traduction du Sūtra du Lotus et son contexte)», in Hanguk chŏntae sasang (Séoul), p. 385-393.

Kıм, Yun-Su (tr.), 2009, Chŏntae Ziūi'ūi Bŏphwa mun'gu'e ūihan myobŏp yŏnhwa'gyŏng (Le Sūtra du Lotus selon le Fahua wenju de Zhiyi), Kwangju.

KIM, Yong-Tae, 1977, «Bŏphwa sin'ang'ūi jŏnraewa gū jŏn'gae (La transmission et le développement de la foi dans [le sūtra de] fleur de la loi)», in Hanguk bulgyohak 3 (Séoul), p. 15-47.

Ko, Ik-Chin, 1987, Hanguk sŏnsul bulsŏ'ūi yŏn'gu (Étude sur les textes bouddhiques coréens sélectionnés), Séoul.

KoHLER, Werner, 1962, Die Lotus-Lehre und die modernene Religionen in Japan, Zürich.

Kwon, Yon-Ung, 1993, «Sejodae'ūi bulgyo jŏngchaek (La politique à l'égard du bouddhisme sous le règne du roi Sejo)», Jindan hakbo 75 (Séoul), p. 197-218.

KYONG'IL, 1987, «Jogyejong'ūi sŏngripsajŏg chūg'myŏn'esŏ bon Bojo (Bojo dans l'histoire de fondation de l'école Jogye)», Bojo sasang, I (Séoul) p. 51-73.

Lamotte, Etienne, 1958, Histoire du bouddhisme indien. Dès origine à l'ère Śaka, Louvain. 
LEE, Dong-Jun, 1981, «Sejong daewang'ūi jŏngūm changjewa hohak jŏngsin (La création du hunmin jŏngūm du roi Sejong et son amour de l'étude)», in Dowa in'gan gwahak, Séoul, p. 187-197.

LEE, Eun-Jeung, 2005, «Historische Entwicklung Koreas», in Südkorea und Nordkorea. Einführung in Geschichte, Politik, Wirtschaft und Gesellschaft, Frankfurt a. M.-New York, p. 15-49.

LEE, Yong-Ja, 1998, Hanguk chŏntae sasang'ūi jŏn'gae (Le développement de la pensée de la Terrasse céleste coréenne), Séoul.

LEe, Yong-Ja, 2003, «A Study on the Lotus Sūtra in India, China and Korea», in S. R. Bhatт (éd.), Buddhist Thought and Culture in India and Korea, New Dehli, p. 96-152.

LI, Ogg, 1969, Histoire de la Corée, Paris.

Magnin, Paul, 2003, Bouddhisme, unité et diversité. Expérience de libération, Paris.

MALMQuist, Göran, 1968-1972, «Die Religionen Chinas», in J. P. AsMUSSEN et J. LAEssøE (éd.), Handbuch der Religionsgeschichte, vol. 3, Göttingen, p. 1-68.

Maruyama, Takao, 1996, «Bŏphwa'gyŏnglon'ūi ipjang (La position du Fahuajing lun)», in C.-S. CHA (tr.), Bŏphwa sasang, Séoul, p. 179-201.

Maspero, Henri, 1950, Les religions chinoises, Paris.

Moтотsuki, Ryōkō, 1996, «Bŏphwa'gyŏng sŏngripsa (Histoire de formation du Sūtra du Lotus)», in C.-S. CHA (tr.), Bŏphwa sasang, Séoul, p. 57-84.

Nomura, Yōshō, 1996, «Ilbulsūng sasang (La pensée du véhicule unique)», in C.-S. CHA (tr.), Bŏphwa sasang, Séoul, p. 133-153.

PARK, Sung-Bae, 1999, «Silla Buddhist Spirituality», in T. YoshinORI (éd.), Buddhist Spirituality. Later China, Korea, Japan and the Modern World, New York, p. 57-78.

Petzold, Bruno, 1987, Die Quintessenz der T'ien-t'ai-(Tendai-)Lehre, Wiesbaden.

Przyluski, Jean, 1932, Le Bouddhisme, Paris.

Renou, Louis et Filliozat, Jean, 1953, L'Inde Classique. Manuel des études indiennes, tome II, (rééd. Paris, 1996).

RHI, Ki-Yong, 2003, «Buddhism and National Culture in Korea», in S. R. BнAтt (éd.), Buddhist Thought and Culture in India and Korea, New Dehli, p. 45-55.

Robert, Jean-Noël, 1993, «Le Grand Véhicule en Inde et en ExtrêmeOrient », in J. Delumeau (dir.), Le fait religieux, Paris, p. 499-529.

Robert, Jean-Noël (tr.), 1997a, Le Sūtra du Lotus. Suivi du Livre des sens incomparables et du Livre de la contemplation de Sage-Universel, Paris. 
RoBert, Jean-Noël, 1997b, «Cinq et cinq font-ils dix ? La double liste des dix ainsités du Sūtra du Lotus », in Le Vase de béryl. Études sur le Japon et la Chine en hommage à Bernard Frank, Paris, p. 35-45.

RoBert, Jean-Noël (tr.), 2007, Quatre courts traités sur la Terrasse Céleste, Paris.

RoBERT, Jean-Noël, «Mahāyāna ou Grand Véhicule», in Encyclopaedia Universalis (version électronique).

SHIOARI, Yodō, 1996, «Chŏntae Ziūi' ūi bŏphwa'gyŏng gwan (La compréhension du Sūtra du Lotus selon Tiantai Zhiyi)», in T.-S. TcHA (tr.), Bŏphwa sasang, Séoul, p. 227-254.

Sørensen, Henrik H., 1999, «Buddhist Spirituality in Premodern and Modern Korea », in Y. TAKEUCHI (éd.), Buddhist Spirituality. Later China, Korea, Japan, and the Modern World, New York, p. 109-133.

SuH, Song-U, 1997, Bŏphwa'gyŏng yŏn'gu (Étude sur le Sūtra du Lotus), Séoul.

Tamura, Yoshiro, 1989, Chŏntae bŏphwa'ūi sasang (Pensée de la Terrasse céleste et [du Sūtra] de fleur de la loi), Y.-J. LEE (tr.), Séoul.

Tsuкамото, Keisho, 2010, Bŏphwa'gyŏng'üi sŏngripgwa bae'gyŏng (Formation du Sūtra du Lotus et son contexte), J.-S. LEE (tr.), Séoul.

UH, Je-Son, 2006, «Bŏphwa'gyŏng'ūi bŏmŏ sabon'gwa hyŏndae hakgye'esǒ'ūi yŏn'gu donghyang (Les manuscrits sanskrits du Sūtra du Lotus et la tendance actuelle de la recherche)», Chŏntaehak yŏn'gu 9 (Séoul), p. 81-98.

VAllÉe Poussin, Louis DE LA, 1909, Bouddhisme. Opinions sur l'Histoire de la Dogmatique, Paris.

Vos, Frits, 1977, Die Religionen Koreas, Stuttgart-Berlin-Köln-Mainz.

Wright, Arthur F., 1959, Buddhism in Chinese History, London.

ZüRCHER, Erik, 1959, The Buddhist Conquest of China. The spread and adaptation of Buddhism in early medieval China, Leiden. 


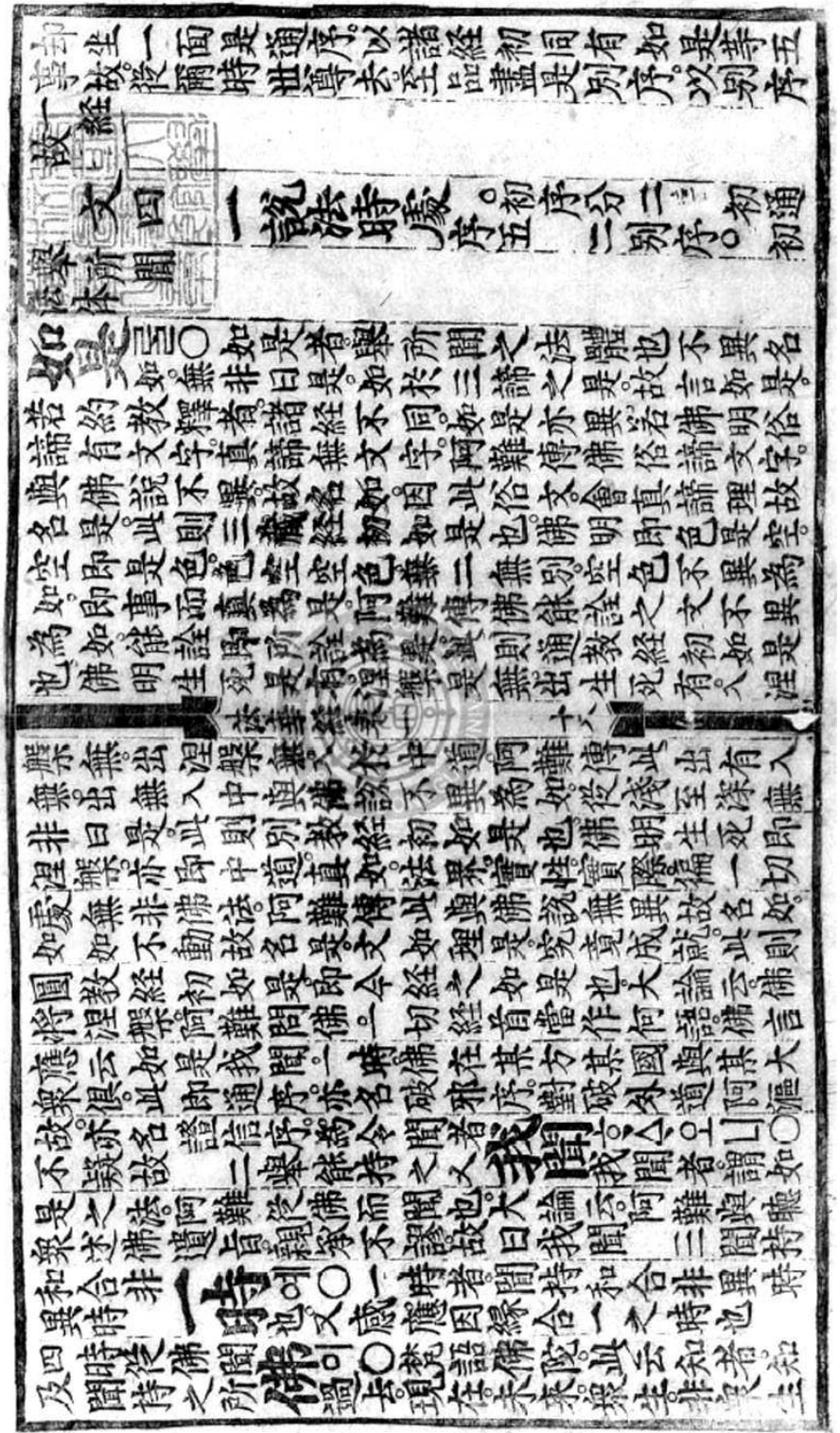

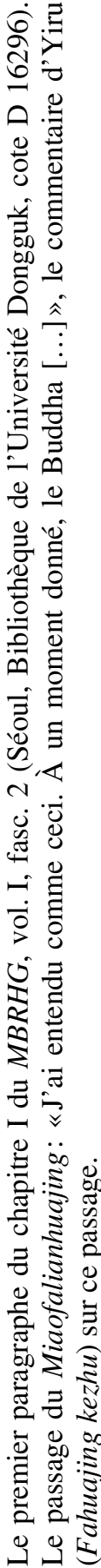




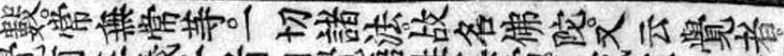

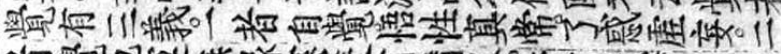

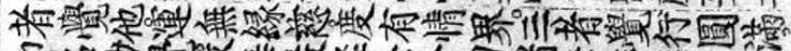

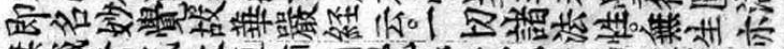

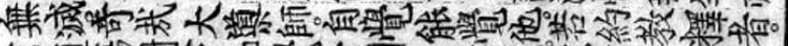

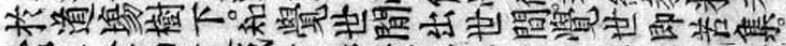

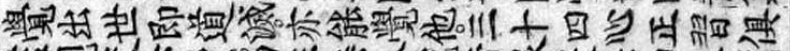

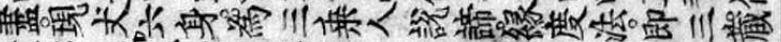

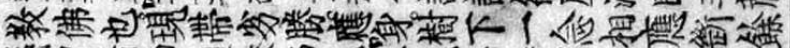
你

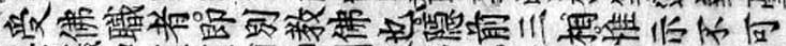

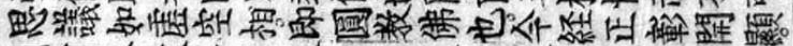

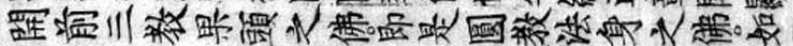

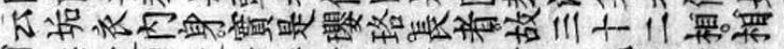

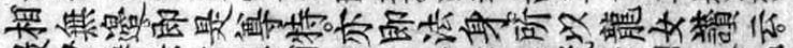

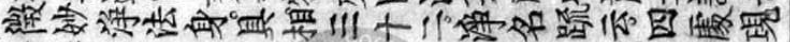

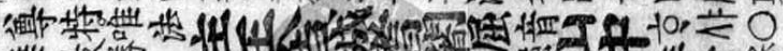

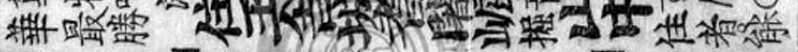

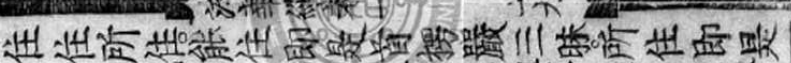

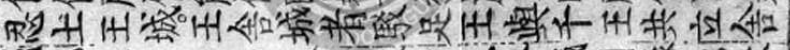

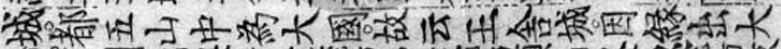

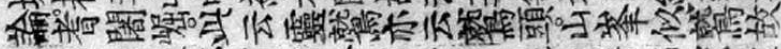

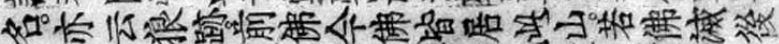

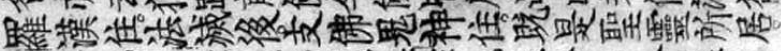

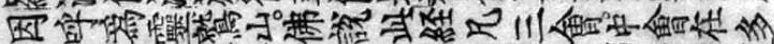

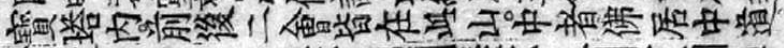
यt

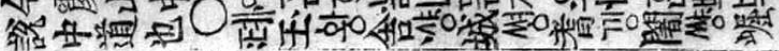

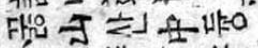

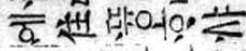

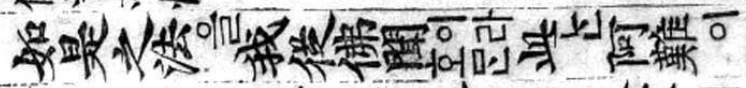

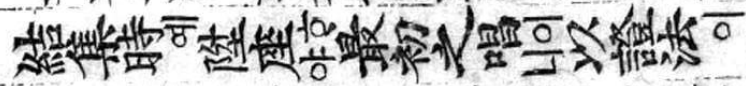

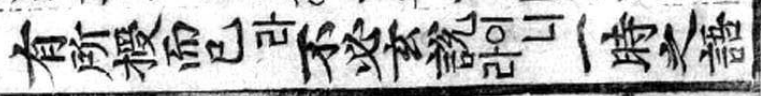




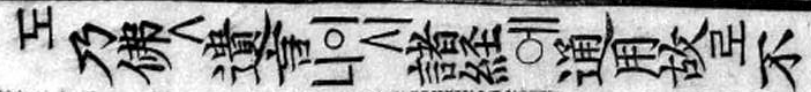

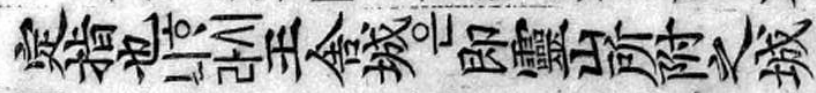

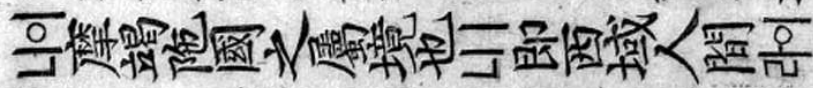

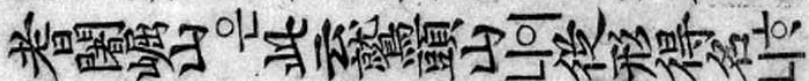

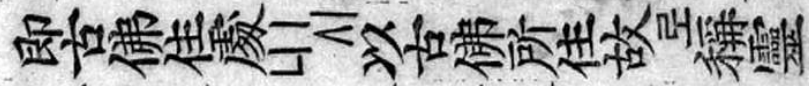

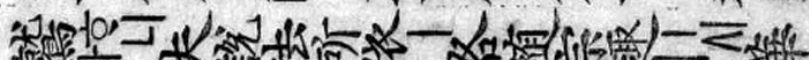

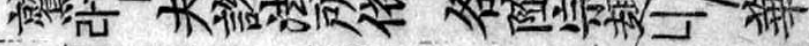

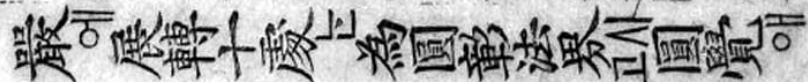

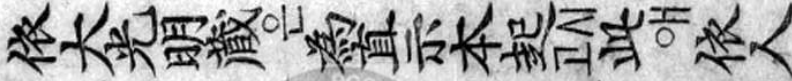

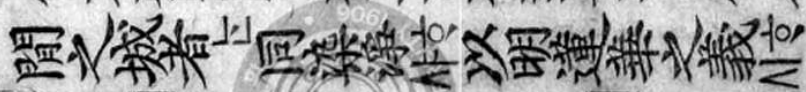
工

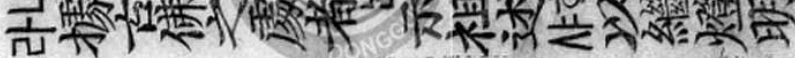

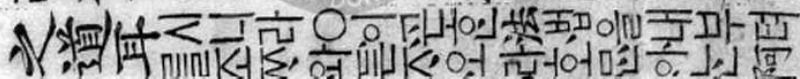

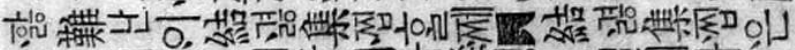

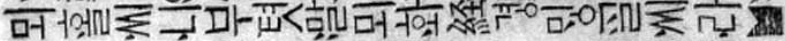

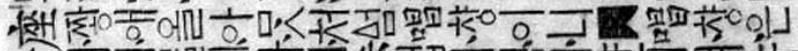

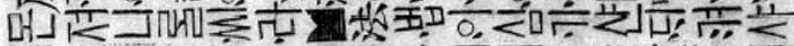

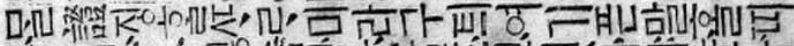

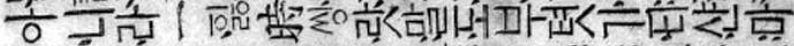

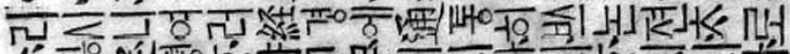

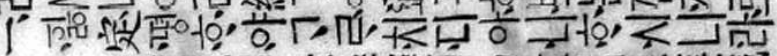

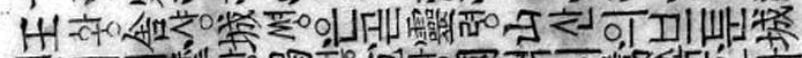

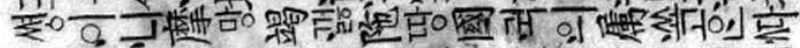

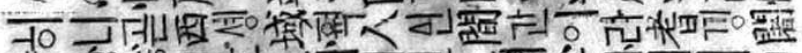

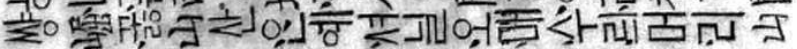

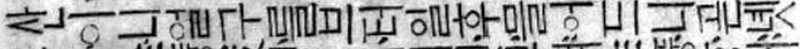

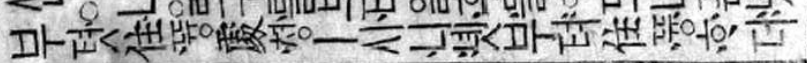

8

ठํㅇ

空

芒

造

그

:ึ :

$\rightarrow 8$

के के

$>$ :

ن

这

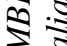

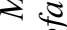

후 :

妾

牲

츨

$\checkmark$.

쿠

0

을

즘

전

ฝี

ธั)

즐

एँ

这.气 